\title{
Hydrodynamic Limit of Condensing Two-Species Zero Range Processes with Sub-critical Initial Profiles
}

\author{
Nicolas Dirr $^{1}$ (D) Marios G. Stamatakis ${ }^{2}$. \\ Johannes Zimmer ${ }^{2}$ (D)
}

Received: 20 October 2016 / Accepted: 21 June 2017 / Published online: 1 July 2017

(C) The Author(s) 2017. This article is an open access publication

\begin{abstract}
Two-species condensing zero range processes (ZRPs) are interacting particle systems with two species of particles and zero range interaction exhibiting phase separation outside a domain of sub-critical densities. We prove the hydrodynamic limit of nearest neighbour mean zero two-species condensing ZRP with bounded local jump rate for sub-critical initial profiles, i.e., for initial profiles whose image is contained in the region of sub-critical densities. The proof is based on H.T. Yau's relative entropy method, which relies on the existence of sufficiently regular solutions to the hydrodynamic equation. In the particular case of the species-blind ZRP, we prove that the solutions of the hydrodynamic equation exist globally in time and thus the hydrodynamic limit is valid for all times.
\end{abstract}

Keywords Hydrodynamic limit - Zero range processes - Condensing zero range processes · Comparison principles for systems of PDEs

\section{Introduction}

In this article, we derive the hydrodynamic limit of a system of two interacting particle systems, specifically two-species zero range processes (ZRPs). The motivation for this study is that hydrodynamic limits provide effective descriptions of large scale interacting particle systems. There is a now good understanding of this limit passage for a range of particle processes leading to one hydrodynamic limit equation. In particular, Kipnis and Landim [19] estab-

$凶$ Nicolas Dirr

DirrNP@cardiff.ac.uk

Marios G. Stamatakis

zimmer@maths.bath.ac.uk

Johannes Zimmer

M.G.Stamatakis@bath.ac.uk; ma.ge.stams@gmail.com

1 School of Mathematics, Cardiff University, Cardiff CF24 4AG, UK

2 Department of Mathematical Sciences, University of Bath, Bath BA2 7AY, UK 
lish the hydrodynamic behaviour for the one-species zero range process, using the entropy method of Guo, Papanicolaou and Varadhan [17]. For systems, however, this limit passage is less well studied, and several tools available for single equations are no longer available, as explained in more detail below. In particular, many systems where a hydrodynamic passage would be of interest both in its own right and as a tool to understand the limiting system of partial differential equations (PDEs) are currently inaccessible to the methods available; the full Patlak Keller-Segel system [18] modelling the evolution of cells or bacteria guided by the concentration of a chemical substance is an example. Yet, there are several recent studies focusing on different models of interacting particle systems. One avenue is to derive equations which incorporate aspects of underlying models, be it by considering the motion of cells only in a stationary, but random, environment mimicking the chemical [14], or by an equation with a singular potential related to a Green's function describing the solution of a second equation [11]. The hydrodynamic limit system of an active exclusion process modelling active matter has been recently derived using a two-block estimate and non-gradient estimates [3].

Another approach is to study systems related to underlying zero-range processes (ZRPs) of several species to obtain a limiting system, and this is the approach we pursue here. The focus on ZRPs can be motivated by their nature as a toy model of an interacting particle system. We consider a system of two zero-range processes but the extension to $n$ types is straightforward. Each ZRP is a process on a lattice where particles jump from one site to another according to a jump rate function depending on the number of the two species of particles on this site only (hence the name zero range).

The hydrodynamic limit in the Eulerian scaling $t \mapsto t N$ of asymmetric many-species ZRPs with product and translation invariant equilibrium states has been studied in [16]. The hydrodynamic limit in the parabolic scaling $t \mapsto t N^{2}$ for a class of processes not satisfying the assumptions of [16] has also recently been studied [26]; there one type of particles performs a random walk and influences the other type, which is a process of ZRP type. In general, establishing hydrodynamic limits for systems of equations rigorously is a hard problem, with few known results so far. To name a few, the hydrodynamic limit of a two-species simple-exclusion process was first studied in [21], the Leroux system has been derived as a hydrodynamic limit in [24], and hyperbolic systems have also been studied in [25].

Here we consider a system of two ZRPs. We show that the hydrodynamic equation is a quasilinear parabolic system of the form

$$
\partial_{t} \rho=\Delta \boldsymbol{\Phi}(\boldsymbol{\rho}), \quad \boldsymbol{\rho}=\left(\rho_{1}, \rho_{2}\right):[0, T) \times \mathbb{T}^{d} \rightarrow A \subseteq \mathbb{R}_{+}^{2},
$$

where $\partial_{t} \rho:=\left(\partial_{t} \rho_{1}, \partial_{t} \rho_{2}\right), \Delta \boldsymbol{\Phi}(\boldsymbol{\rho}):=\left(\Delta \Phi_{1}(\rho), \Delta \Phi_{2}(\rho)\right)$ and $\boldsymbol{\Phi}: A \rightarrow \mathbb{R}_{+}^{2}$ is the mean jump rate of the ZRP at a site $x \in \mathbb{T}_{N}^{d}$ under the product and translation invariant equilibrium state of background density $\rho \in A \subseteq \mathbb{R}_{+}^{2}$. Two-species ZRPs, and the phase transition they exhibit, were first studied in [8]. In condensing ZRPs, the set $A$ of admissible background densities $\rho$ is a strict subset of $\mathbb{R}_{+}^{2}$. We call such densities sub-critical.

One challenge of ZRPs is that they can exhibit condensation phenomena, where particles congregate at the same site $[7,9,15]$. Even for one-species systems, the hydrodynamic limit of ZRPs experiencing condensation is presently unknown. We consider parameter regimes of two-species systems where condensation can occur, but restrict to sub-critical initial profiles, i.e., initial data that take values in the set of sub-critical densities. For one-species ZRPs, the analogous result has been established recently [23] and we extend this argument to the two-species case. Specifically, we apply the relative entropy method of H. T. Yau [28], 
which requires only the one-block estimate proved in Theorem 3.1, not the full replacement lemma [19, Lemma 5.1.10]. Thus it does not require the equilibrium states of the ZRP to have full exponential moments, a property not satisfied by condensing ZRPs. This extension of [23] is non-trivial, for two reasons. The first difficulty is that the relative entropy method requires the existence of $C^{2+\theta}$ solutions to (1) for some $\theta \in(0,1]$, and that the solution remains in the sub-critical region. By a result of Amann [1] it is know that, when starting from $C^{2+\theta}$ initial data, $C^{1,2+\theta}$ solutions of uniformly parabolic systems exist locally in time, i.e., for small time intervals, and are unique. Thus there exists a unique maximally defined classical solution of the parabolic system (1) taking values in the sub-critical region $A$. So our general result on the hydrodynamic limit is local in time, being valid for the largest time interval for which the unique maximal classical $C^{1,2+\theta}$ solution with values in the sub-critical region exists. This result shows that at least for as long as the unique maximal classical solution to the parabolic system (1) is defined, condensation does not occur. The second difficulty to extend the results for one species $[19,23]$ is that the phase space $\mathbb{R}_{+}^{2}$ is now more complicated, and the one-dimensional arguments used in $[19,23]$ do not extend directly. In particular, a novel argument is required to extend [19, Lemma 6.1.10]; see Lemma 4.5 and its proof. Specifically, we employ a characterisation of the domain of a convex function via the recession function of its Legendre transform. This characterisation of the domain of convex functions is of interest in its own right in the context of two-species ZRPs. For example, it immediately yields a parametrisation of the boundary of the domain of the partition function via the recession function of the thermodynamic entropy.

Intuitively, condensation means on the level of the governing hydrodynamic limit PDE the formation of singularities where the mass concentrates. For scalar equations, the formation of such singularities can be ruled out by a maximum principle. For systems, however, in general maximum principles do not hold. In this article, we mainly rely on an existence theory for local $C^{1,2+\theta}$ classical solutions established by Amann and focus on proving the local in time hydrodynamic limit. However, for a particular example, the so-called species-blind process, we are able to establish a maximum principle and $C^{1+\theta, 2+\theta}$ regularity for the hydrodynamic equation. This allows us to obtain that $C^{1+\theta, 2+\theta}$ solutions exist and remain in the sub-critical region for all times. So in this particular case, the result on the hydrodynamic limit is global in time.

Maximum principles are more complicated for non-linear parabolic systems, since one has to determine the shape of the invariant region in which the solution will have to remain [6,27], while in the scalar case the invariant region is just an interval. For the species-blind process we find that the invariant region of the hydrodynamic equation coincides with the sub-critical region of the ZRP. This is not surprising since the species-blind process is obtained from a one-species ZRP by colouring particles in two colours, say black and white. The dynamics is the usual ZRP dynamics but at each time of a jump from a site $x$, we choose the colour of particle to move with the probabilities given by the ratios of the number of black particles and white particles at $x$ to the total number of particles at $x$, ignoring the colour. It would still be interesting to study the class of parabolic systems arising from two-species ZRPs in order to determine the largest class of ZRPs that their sub-critical region is an invariant region of the hydrodynamic limit. This would then provide a way to find the invariant region of the associated parabolic systems by calculating the phase diagram of the underlying ZRP. The study of the system of PDEs arising from the ZRP is a different topic and outside of the scope of this article, which mainly focuses on the passage from the microscopic to the macroscopic level by applying the relative entropy method. 


\subsection{Plan of the Paper}

The paper is organised as follows. In Sect. 2 we collect some preliminary material on twospecies ZRPs and describe the particular case of the species-blind ZRP. Section 3 contains the statements of the main results, and in Sect. 4 we give the proofs.

\section{The Particle Model}

We briefly give the definition of two-species ZRPs as Markov jump processes (Sect. A.1.2 in [19]) and their equilibrium states. Main references on this preliminary material are [12,13]. We take the discrete $d$-dimensional $N$-torus $\mathbb{T}_{N}^{d}$ as underlying lattice. Each particle interacts only with particles in the same lattice site through a function $\boldsymbol{g}=\left(g_{1}, g_{2}\right): \mathbb{N}_{0}^{2} \rightarrow \mathbb{R}_{+}^{2}$. Here $g_{i}(\boldsymbol{k})$ is the jump rate of species of type $i$ from any site that contains $\boldsymbol{k} \in \mathbb{N}_{0}^{2}$ particles, i.e., $k_{i}$ particles of type $i$, for $i=1,2$. We impose the natural condition

$$
g_{i}(k)=0 \quad \text { iff } k_{i}=0, \quad \boldsymbol{k}=\left(k_{1}, k_{2}\right) \in \mathbb{N}_{0}^{2}
$$

and require

$$
\left\|\partial_{i} g_{i}\right\|_{\infty}:=\sup _{\boldsymbol{k} \in \mathbb{N}_{0}^{2}}\left|g_{i}\left(\boldsymbol{k}+\boldsymbol{e}_{i}\right)-g_{i}(\boldsymbol{k})\right|<+\infty,
$$

where $\boldsymbol{e}_{i}=\left(\delta_{i j}\right)_{j=1,2}, i=1,2$, are the unit vectors in $\mathbb{R}^{2}$. Note that setting $\boldsymbol{g}^{*}:=\left\|\partial_{1} g_{1}\right\|_{\infty} \vee$ $\left\|\partial_{2} g_{2}\right\|_{\infty}<+\infty$, we have by (2) and (3) that

$$
|\boldsymbol{g}(\boldsymbol{k})|_{p} \leq \boldsymbol{g}^{*}|\boldsymbol{k}|_{p}, \text { for every } \boldsymbol{k} \in \mathbb{N}_{0}^{2},
$$

where $|\cdot|_{p}$ denotes the $\ell_{p}$-norm in $\mathbb{R}^{2}, p \in[1,+\infty]$.

The state space of a two-species ZRP consists of all configurations $\boldsymbol{\eta}=\left(\eta_{1}, \eta_{2}\right): \mathbb{T}_{N}^{d} \rightarrow$ $\mathbb{N}_{0}^{2}$, so that $\eta_{i}(x)$ is the number of $i$-type particles at site $x$, for $i=1,2$. For any measurable space $M$ we denote by $\mathbb{P}(M)$ the set of all probability measures on $M$. We write $p \in \mathbb{P}\left(\mathbb{Z}^{d}\right)$ for the nearest neighbour (n.n.) elementary step distribution given by

$$
p(x):=\frac{1}{2 d} \sum_{j=1}^{d} \mathbb{1}_{\left\{-e_{j}, e_{j}\right\}}(x), \quad x \in \mathbb{Z}^{d},
$$

and by $p_{N} \in \mathbb{P}\left(\mathbb{T}_{N}^{d}\right)$ its projection on $\mathbb{T}_{N}^{d}$ given by $p_{N}(x):=p\left(x+N \mathbb{Z}^{d}\right)$. Also, given a configuration $\eta \in \mathbb{M}_{N}^{d ; 2}:=\left(\mathbb{N}_{0}^{2}\right)^{\mathbb{T}_{N}^{d}}$, we will denote by $\eta^{i ; x ; y}, i=1,2$, the configuration resulting from $\eta$ by moving a type-i particle from $x$ to $y$. (If $\eta_{i}(x)=0$, then we set $\eta^{i ; x ; y}=$ $\eta$.) The two-species n.n. symmetric ZRP with jump rate $\boldsymbol{g}$ on the discrete torus $\mathbb{T}_{N}^{d}:=$ $\{0,1, \ldots, N-1\}^{d}$ is the unique Markov jump process on the Skorohod space $D\left(\mathbb{R}_{+} ; \mathbb{M}_{N}^{d ; 2}\right)$ of càdlàg paths characterised by the formal generator

$$
L^{N} f(\boldsymbol{\eta})=\sum_{i=1,2} \sum_{x, y \in \mathbb{T}_{N}^{d}}\left\{f\left(\boldsymbol{\eta}^{i: x, y}\right)-f(\boldsymbol{\eta})\right\} g_{i}(\boldsymbol{\eta}(x)) p_{N}(y-x) .
$$

We will denote by $\left(P_{t}^{N}\right)_{t \geq 0}$ the transition semigroup of the n.n. symmetric ZRP. The communication classes of the stochastic dynamics defined by the generator above are the hyperplanes

$$
\mathbb{M}_{N, \boldsymbol{K}}^{d ; 2}:=\left\{\eta \in \mathbb{M}_{N}^{d ; 2} \mid \sum_{x \in \mathbb{T}_{N}^{d}} \eta(x)=\boldsymbol{K}\right\}
$$


consisting of a fixed number of particles of each species. Since each set $\mathbb{M}_{N, K}^{d ; 2}$ is finite, for each $(N, \boldsymbol{K}) \in \mathbb{N} \times \mathbb{N}_{0}^{2}$ there exists a unique equilibrium distribution $v_{N, \boldsymbol{K}}$ supported on $\mathbb{M}_{N, \boldsymbol{K}}^{d ; 2}$. The family $\left\{v_{N, \boldsymbol{K}}\right\}_{(N, \boldsymbol{K}) \in \mathbb{N} \times \mathbb{N}_{0}^{2}}$ is the so-called canonical ensemble. However, as proved in [12, Theorem 4.1], in order to have product and translation invariant equilibrium distributions, it is necessary and sufficient that the following compatibility relations for the component functions of two-species jump rates hold,

$$
g_{1}(\boldsymbol{k}) g_{2}\left(\boldsymbol{k}-\boldsymbol{e}_{1}\right)=g_{1}\left(\boldsymbol{k}-\boldsymbol{e}_{2}\right) g_{2}(\boldsymbol{k}), \quad \text { for all } \boldsymbol{k} \in \mathbb{N}_{0}^{2} \text { with } k_{1}, k_{2} \geq 1 .
$$

Note that due to the compatibility relations (6) any two-species local jump rate $g$ is uniquely determined by $g_{1}$ and the restriction of $g_{2}$ to the set $\{0\} \times \mathbb{N}_{0}$, since by induction for any $\boldsymbol{k} \in \mathbb{N}_{0}^{2}$

$$
g_{2}(\boldsymbol{k})=g_{2}\left(0, k_{2}\right) \prod_{i=0}^{k_{1}-1} \frac{g_{1}\left(\boldsymbol{k}-i \boldsymbol{e}_{1}\right)}{g_{1}\left(\boldsymbol{k}-i \boldsymbol{e}_{1}-\boldsymbol{e}_{2}\right)} .
$$

An increasing path $\boldsymbol{\gamma}$ (from 0 ) to $\boldsymbol{k} \in \mathbb{N}_{0}^{2}$ is any path $\boldsymbol{\gamma}:\left\{0, \ldots, k_{1}+k_{2}\right\} \rightarrow \mathbb{N}_{0}^{2}$ such that $\boldsymbol{\gamma}(0)=0, \boldsymbol{\gamma}\left(k_{1}+k_{2}\right)=\boldsymbol{k}$ and $\boldsymbol{\gamma}(\ell)=\boldsymbol{\gamma}(\ell-1)+\boldsymbol{e}_{i_{\ell}}$ for some $i_{\ell} \in\{1,2\}$ for all $\ell=1, \ldots, k_{1}+k_{2}$. For any increasing path $\gamma$ to $\boldsymbol{k} \in \mathbb{N}_{0}^{2}$, the factorial of $\boldsymbol{g}$ along $\boldsymbol{\gamma}$ is defined as

$$
\boldsymbol{g} !(\boldsymbol{k} ; \boldsymbol{\gamma})=\prod_{\ell=1}^{k_{1}+k_{2}} g_{i_{\ell}}(\boldsymbol{\gamma}(\ell))
$$

for $\boldsymbol{k} \neq 0$; we set $\boldsymbol{g} !(\cdot):=1$ if $\boldsymbol{k}=0$. A two-species local jump rate function $\boldsymbol{g}$ that satisfies (6) yields a well-defined function $g$ !: $\mathbb{N}_{0}^{2} \rightarrow(0, \infty)$ by the formula

$$
\boldsymbol{g} !(\boldsymbol{k})=\boldsymbol{g} !(\boldsymbol{k} ; \boldsymbol{\gamma}) \quad \text { for some increasing path } \boldsymbol{\gamma} \text { to } \boldsymbol{k} .
$$

For instance

$$
\begin{aligned}
\boldsymbol{g} !(\boldsymbol{k}) & =g_{1}(1,0) \cdot \ldots \cdot g_{1}\left(k_{1}, 0\right) \cdot g_{2}\left(k_{1}, 1\right) \cdot \ldots \cdot g_{2}\left(k_{1}, k_{2}\right) \\
& =g_{2}(0,1) \cdot \ldots \cdot g_{2}\left(0, k_{2}\right) \cdot g_{1}\left(1, k_{2}\right) \cdot \ldots \cdot g_{1}\left(k_{1}, k_{2}\right) .
\end{aligned}
$$

According to [12, Theorem 4.1], using the multi-index notation $\varphi^{k}:=\varphi_{1}^{k_{1}} \varphi_{2}^{k_{2}}$, with $\boldsymbol{\varphi}, \boldsymbol{k} \in \mathbb{R}_{+}^{2}$, for two-species symmetric n.n. ZRP satisfying (6), the common one-site marginal $\bar{v}_{\varphi}^{1}$ of the product and translation invariant equilibrium states $\bar{v}_{\varphi}^{N}$ is given by the formula

$$
\bar{v}_{\varphi}^{1}(k)=\frac{1}{Z(\varphi)} \frac{\varphi^{k}}{g !(k)}, \quad k \in \mathbb{N}_{0}^{2}
$$

for all $\varphi \in \mathbb{R}_{+}^{2}$ such that the series

$$
Z(\varphi):=\sum_{k \in \mathbb{N}_{0}^{2}} \frac{\varphi^{k}}{g !(k)}
$$

converges. The function $Z: \mathbb{R}_{+}^{2} \rightarrow[1,+\infty]$ defined in (7) is called the partition function. The main convexity property of $Z$ is that the function $\mathcal{Z}:=Z \circ \exp : \mathbb{R}^{2} \rightarrow(1,+\infty]$ is strictly $\operatorname{logarithmically}$ convex where $\exp (\boldsymbol{\mu}):=e^{\mu}:=\left(e^{\mu_{1}}, e^{\mu_{2}}\right)$. This can be seen by applying Hölder's inequality to the functions $\boldsymbol{k} \mapsto e^{\langle\boldsymbol{\mu}, \boldsymbol{k}\rangle}, \boldsymbol{k} \mapsto e^{\langle\boldsymbol{v}, \boldsymbol{k}\rangle}$ with respect to the 
$\sigma$-finite measure $\lambda$ on $\mathbb{N}_{0}^{2}$ given by $\lambda(\boldsymbol{k}):=\frac{1}{g !(\boldsymbol{k})}$ and with the pair of conjugate exponents $p=\frac{1}{1-t}, q=\frac{1}{t}$ for $t \in(0,1)$ and $\boldsymbol{\mu}, \boldsymbol{v} \in \mathbb{R}^{2}$ such that $\boldsymbol{\mu} \neq \boldsymbol{v}$, which yields

$$
\mathcal{Z}((1-t) \boldsymbol{\mu}+t \boldsymbol{v})=\int e^{(1-t)\langle\boldsymbol{\mu}, \boldsymbol{k}\rangle} e^{t\langle\boldsymbol{v}, \boldsymbol{k}\rangle} d \lambda(\boldsymbol{k})<\mathcal{Z}(\boldsymbol{\mu})^{(1-t)} \mathcal{Z}(\boldsymbol{v})^{t} .
$$

Here and in what follows $\langle\boldsymbol{\mu}, \boldsymbol{k}\rangle=\mu_{1} k_{1}+\mu_{2} k_{2}$ denotes the Euclidean inner product of two vectors $\boldsymbol{k}, \boldsymbol{\mu} \in \mathbb{R}_{+}^{2}$. We denote by $\mathcal{D}_{Z}:=\left\{\boldsymbol{\varphi} \in \mathbb{R}_{+}^{2} \mid Z(\varphi)<+\infty\right\}$ the proper domain of $Z$, which is a complete, i.e., $[0, \varphi]:=\left[0, \varphi_{1}\right] \times\left[0, \varphi_{2}\right] \subseteq \mathcal{D}_{Z}$ for all $\varphi \in \mathcal{D}_{Z}$, and logarithmically convex set, that, is the set $\mathcal{D}_{\mathcal{Z}}=\log \left(\mathcal{D}_{Z} \cap(0, \infty)^{2}\right):=\left\{\log \varphi:=\left(\log \varphi_{1}, \log \varphi_{2}\right) \mid \varphi \in\right.$ $\left.\mathcal{D}_{Z} \cap(0, \infty)^{2}\right\}$ is convex. The partition function is $C^{\infty}$ in $\mathcal{D}_{Z}^{o}$ and continuous from below on $\mathcal{D}_{Z}$, i.e., for all $\varphi \in \mathcal{D}_{Z}, \varepsilon>0$ there exists $\delta>0$ such that $|Z(\varphi)-Z(\boldsymbol{\psi})|<\varepsilon$ for all $\psi \in D(0, \delta) \cap[0, \varphi]$. Here $D(0, \delta)$ denotes the Euclidean open ball of radius $\delta$ with centre 0 in $\mathbb{R}_{+}^{2}$, i.e., $D(0, \delta)=\left\{\left.\varphi \in \mathbb{R}_{+}^{2}|| \varphi\right|_{2}<\delta\right\}$.

The family of the product and translation invariant equilibrium states is the family $\left\{\bar{v}_{\varphi}^{N}\right\}_{\varphi \in \mathcal{D}_{Z}}$. This family is usually referred to as the grand canonical ensemble (GCE). In order to ensure that $\mathcal{D}_{Z}$ is not trivial, i.e., that $\mathcal{D}_{Z}$ contains a neighbourhood of zero in $\mathbb{R}_{+}^{2}$, we must impose the following condition in the definition of two-species local jump rate functions:

$$
\varphi_{* ; 1}:=\liminf _{|\boldsymbol{k}|_{1} \rightarrow+\infty} \boldsymbol{g} !(\boldsymbol{k})^{\frac{1}{\left.k\right|_{1}}}>0 .
$$

A two-species local jump rate $\boldsymbol{g}$ satisfies (8) iff $\mathcal{D}_{Z}$ contains a neighbourhood of 0 in $\mathbb{R}_{+}^{2}$. In what follows, we consider only two-species local jump rates that satisfy (6) and (8).

It is convenient to have a parametrisation of the GCE by the density. This is done via the density function $\boldsymbol{R}=\left(R_{1}, R_{2}\right): \mathcal{D}_{Z} \rightarrow[0,+\infty]^{2}$ defined by

$$
\boldsymbol{R}(\boldsymbol{\varphi})=\int_{\mathbb{M}_{N}^{d ; 2}} \boldsymbol{\eta}(0) d \bar{v}_{\varphi}^{N}=\left(\int k_{1} d \bar{\nu}_{\varphi}^{1}, \int k_{2} d \bar{\nu}_{\varphi}^{1}\right) .
$$

The proper domain of $\boldsymbol{R}$ is the set $\mathcal{D}_{\boldsymbol{R}}:=\left\{\boldsymbol{\varphi} \in D_{Z} \mid \boldsymbol{R}(\boldsymbol{\varphi}) \in \mathbb{R}_{+}^{2}\right\}$ and by differentiation of bivariate power-series, we have that

$$
\boldsymbol{R}(\boldsymbol{\varphi})=\boldsymbol{\varphi} \cdot \nabla(\log Z)(\varphi) \quad \text { on the set } \mathcal{D}_{\boldsymbol{R}}^{o}=\mathcal{D}_{Z}^{o},
$$

where $\boldsymbol{\varphi} \cdot \boldsymbol{\psi}:=\left(\varphi_{1} \psi_{1}, \varphi_{2} \psi_{2}\right)$ denotes the pointwise product of two vectors $\boldsymbol{\varphi}, \boldsymbol{\psi} \in \mathbb{R}_{+}^{2}$. Furthermore, this formula extends to the set $\mathcal{D}_{Z} \cap \partial \mathcal{D}_{Z}$ if we interpret the directional derivatives $\partial_{i}(\log Z) \in[0,+\infty]$ as derivatives from the left. With the conventions $\log 0=-\infty$ and $e^{-\infty}=0$ the densities $\rho \in \boldsymbol{R}\left(\mathcal{D}_{\boldsymbol{R}}\right)$ can also be parametrised via the chemical potential by the function $\mathcal{R}:=\boldsymbol{R} \circ \exp : \mathcal{D}_{\mathcal{R}} \rightarrow \boldsymbol{R}\left(\mathcal{D}_{\boldsymbol{R}}\right)$, where $\mathcal{D}_{\mathcal{R}}=\log \left(\mathcal{D}_{\boldsymbol{R}}\right):=$ $\left\{\log \varphi \in[-\infty,+\infty)^{2} \mid \varphi \in \mathcal{D}_{\boldsymbol{R}}\right\}$. For the parametrisation via the chemical potentials $\mathcal{R}(\boldsymbol{\mu})=\nabla(\log \mathcal{Z})(\boldsymbol{\mu})$ for all $\boldsymbol{\mu} \in \mathcal{D}_{\mathcal{R}}^{o} \cap(-\infty,+\infty)^{2}$, where $\mathcal{Z}=Z$ o exp.

The density function $\boldsymbol{R}: \boldsymbol{R}\left(\mathcal{D}_{\boldsymbol{R}}\right) \rightarrow \mathcal{D}_{\boldsymbol{R}}$ is invertible. Indeed, it is straightforward to check (e.g., see [13, (4.10)]) that for all $\varphi \in \mathcal{D}_{\boldsymbol{R}}^{o} \cap(0,+\infty)^{2}$,

$$
D \boldsymbol{R}(\boldsymbol{\varphi})=D \mathcal{R}(\log \varphi)\left(\begin{array}{cc}
\frac{1}{\varphi_{1}} & 0 \\
0 & \frac{1}{\varphi_{2}}
\end{array}\right)=\operatorname{Cov}\left(\bar{v}_{\varphi}^{1}\right)\left(\begin{array}{cc}
\frac{1}{\varphi_{1}} & 0 \\
0 & \frac{1}{\varphi_{2}}
\end{array}\right),
$$

where $\operatorname{Cov}\left(\bar{v}_{\varphi}^{1}\right)$ denotes the covariance matrix

$$
\operatorname{Cov}\left(\bar{v}_{\varphi}^{1}\right)_{i j}=\int k_{i} k_{j} d \bar{v}_{\varphi}^{1}-\int k_{i} \bar{v}_{\varphi}^{1} \int k_{j} \bar{v}_{\varphi}^{1}, \quad i, j=1,2 .
$$


This implies that $D \boldsymbol{R}(\boldsymbol{\varphi})$ is diagonisable with strictly positive eigenvalues for all $\boldsymbol{\varphi} \in \mathcal{D}_{\boldsymbol{R}}^{o} \cap$ $(0,+\infty)^{2}$. Furthermore,

$$
\partial_{1} R_{1}(\varphi) \wedge \partial_{2} R_{2}(\varphi)>0 \text { for all } \varphi \in \mathcal{D}_{R}^{o}
$$

and for $\boldsymbol{\varphi} \in \mathcal{D}_{\boldsymbol{R}}^{o}$ such that $\varphi_{1} \varphi_{2}=0$, the matrix $D \boldsymbol{R}(\boldsymbol{\varphi})$ is triangular, and thus invertible. Therefore the density function $\boldsymbol{R}: \mathcal{D}_{\boldsymbol{R}}^{o} \rightarrow \boldsymbol{R}\left(\mathcal{D}_{\boldsymbol{R}}^{o}\right)$ is invertible. The fact that $\boldsymbol{R}$ is invertible on all of its domain follows by [13, Proposition 2.3], according to which for every $\rho \in(0, \infty)^{2}$ there exists a unique maximiser $\overline{\boldsymbol{\Phi}}(\boldsymbol{\rho}) \in \mathcal{D}_{\boldsymbol{R}} \cap(0, \infty)^{2}$ for the thermodynamic entropy

$$
S(\boldsymbol{\rho}):=\sup _{\boldsymbol{\varphi} \in \mathcal{D}_{Z} \cap(0, \infty)^{2}}\{\langle\boldsymbol{\rho}, \log \boldsymbol{\varphi}\rangle-\log Z(\varphi)\}=\langle\rho, \log \overline{\boldsymbol{\Phi}}(\boldsymbol{\rho})\rangle-\log Z(\overline{\boldsymbol{\Phi}}(\boldsymbol{\rho})) .
$$

Obviously, for $\rho=0$ the supremum is attained at $\varphi=0$ (with the convention $0 \cdot(-\infty)=0$ ). Furthermore, since $Z$ is non-decreasing with respect to each variable separately, for any $\rho \in \mathbb{R}_{+}^{2} \backslash\{0\}$ with $\rho_{1} \rho_{2}=0$, say $\rho_{2}=0$, the maximisation problem (11) is reduced to the corresponding maximisation problem for one of the one-species jump rate $\hat{g}_{1}(k):=g_{1}(k, 0)$, $k \in \mathbb{N}_{0}$ and the supremum is attained at $\overline{\boldsymbol{\Phi}}\left(\rho_{1}, 0\right)=\left(\hat{\Phi}_{1}\left(\rho_{1} \wedge \hat{\rho}_{c, 1}\right), 0\right)$, where $\hat{\Phi}_{1}, \hat{\rho}_{c, 1}$ are the mean jump rate and critical density of the one-species jump rate $\hat{g}_{1}$ (see [12, Sect. 5.2.1] for the one-species case). Thus for any $\rho \in \mathbb{R}_{+}^{2}$ there exists a unique maximiser $\overline{\boldsymbol{\Phi}}(\rho) \in \mathcal{D}_{\boldsymbol{R}}$ for the thermodynamic entropy $S(\rho)$. As in [13, Proposition 2.3] the function $\boldsymbol{\Phi}: \mathbb{R}_{+}^{2} \rightarrow \mathcal{D}_{\boldsymbol{R}}$ is continuous, $\boldsymbol{\Phi}:=\left.\overline{\boldsymbol{\Phi}}\right|_{\boldsymbol{R}\left(\mathcal{D}_{\boldsymbol{R}}\right)}=\boldsymbol{R}^{-1}$ is the inverse of $\boldsymbol{R}: \mathcal{D}_{\boldsymbol{R}} \rightarrow \boldsymbol{R}\left(\mathcal{D}_{\boldsymbol{R}}\right)$ and

$$
\overline{\boldsymbol{\Phi}}\left(\mathbb{R}_{+}^{2} \backslash \boldsymbol{R}\left(\mathcal{D}_{\boldsymbol{R}}\right)\right)=\mathcal{D}_{\boldsymbol{R}} \cap \partial \mathcal{D}_{\boldsymbol{R}} .
$$

Furthermore $\boldsymbol{R}\left(\mathcal{D}_{\boldsymbol{R}}\right)$ is closed in $\mathbb{R}_{+}^{2}$ and $\partial \boldsymbol{R}\left(\mathcal{D}_{\boldsymbol{R}}\right)=\boldsymbol{R}\left(\mathcal{D}_{\boldsymbol{R}} \cap \partial \mathcal{D}_{\boldsymbol{R}}\right)$. According to this result $\overline{\boldsymbol{\Phi}}: \mathbb{R}_{+}^{2} \rightarrow \mathcal{D}_{\boldsymbol{R}}$ is a left inverse for $\boldsymbol{R}$, i.e., $\overline{\boldsymbol{\Phi}} \circ \boldsymbol{R}=\boldsymbol{\Phi} \circ \boldsymbol{R}=\dot{\mathrm{i}} \mathbb{d}_{\mathcal{D}_{\boldsymbol{R}}}$ and the function

$$
\boldsymbol{R}_{c}:=\boldsymbol{R} \circ \overline{\boldsymbol{\Phi}}: \mathbb{R}_{+}^{2} \rightarrow \boldsymbol{R}\left(\mathcal{D}_{\boldsymbol{R}}\right)
$$

is a continuous projection on $\boldsymbol{R}\left(\mathcal{D}_{\boldsymbol{R}}\right)$ with $\left.\boldsymbol{R}_{C}\right|_{\boldsymbol{R}\left(\mathcal{D}_{\boldsymbol{R}}\right)}=\dot{\mathrm{i}} \mathbb{d}_{\boldsymbol{R}\left(\mathcal{D}_{\boldsymbol{R}}\right)}$, satisfying

$$
\boldsymbol{R}_{c}\left(\mathbb{R}_{+}^{2} \backslash \boldsymbol{R}\left(\mathcal{D}_{\boldsymbol{R}}\right)\right)=\boldsymbol{R}\left(\mathcal{D}_{\boldsymbol{R}} \cap \partial \mathcal{D}_{\boldsymbol{R}}\right)=\partial \boldsymbol{R}\left(\mathcal{D}_{\boldsymbol{R}}\right) .
$$

In particular, $\boldsymbol{R}: \mathcal{D}_{\boldsymbol{R}} \rightarrow \boldsymbol{R}\left(\mathcal{D}_{\boldsymbol{R}}\right)$ is a homeomorphism and $\boldsymbol{R}\left(\mathcal{D}_{\boldsymbol{R}}\right)^{o}=\boldsymbol{R}\left(\mathcal{D}_{\boldsymbol{R}}^{o}\right)$.

Note that the thermodynamic entropy coincides with the Legendre transform of the convex thermodynamic pressure $\log \mathcal{Z}: \mathbb{R}^{2} \rightarrow(0,+\infty]$, that is,

$$
S(\boldsymbol{\rho})=(\log \mathcal{Z})^{*}(\boldsymbol{\rho})=\sup _{\boldsymbol{\mu} \in \mathbb{R}^{2}}\{\langle\boldsymbol{\rho}, \boldsymbol{\mu}\rangle-\log \mathcal{Z}(\boldsymbol{\mu})\} .
$$

Since $\nabla(\log \mathcal{Z})=\mathcal{R}=\boldsymbol{R} \circ \exp$, it follows by the formula for the derivative of the Legendre transforms that for all $\rho \in(0, \infty)^{2} \cap \boldsymbol{R}\left(\mathcal{D}_{\boldsymbol{R}}^{o}\right)$ the supremum in (12) is attained at

$$
\nabla S(\rho)=(\nabla \log \mathcal{Z})^{-1}(\rho)=\mathcal{R}^{-1}(\rho)=\log \Phi(\rho) .
$$

Since $S$ is convex the matrix $D^{2} S(\rho)=D(\log \Phi)(\rho)$ is symmetric and strictly positive definite for all $\rho \in \boldsymbol{R}\left(\mathcal{D}_{\boldsymbol{R}}^{o}\right) \cap(0,+\infty)^{2}$. The symmetry of $D^{2} S(\rho), \rho \in \boldsymbol{R}\left(\mathcal{D}_{\boldsymbol{R}}^{o}\right) \cap(0,+\infty)^{2}$, implies the relations

$$
\Phi_{2}(\rho) \partial_{2} \Phi_{1}(\rho)=\Phi_{1}(\rho) \partial_{1} \Phi_{2}(\rho),
$$

which extend to $\rho \in \boldsymbol{R}\left(\mathcal{D}_{\boldsymbol{R}}^{o}\right)$ because $R_{i}(\boldsymbol{\varphi})=0$ if and only if $\varphi_{i}=0, i=1,2$ and $D \boldsymbol{R}(\boldsymbol{\varphi})$ is triangular for $\boldsymbol{\varphi} \in \mathcal{D}_{\boldsymbol{R}}^{o}$ with $\varphi_{1} \varphi_{2}=0$. Equation (13) can be seen as the macroscopic analogue of the compatibility relations (6). 
Using the inverse $\boldsymbol{\Phi}$ of $\boldsymbol{R}$ on $\boldsymbol{R}\left(\mathcal{D}_{\boldsymbol{R}}\right)$, we can parametrise the grand canonical measures $\bar{v}_{\varphi}^{N}, \varphi \in \mathcal{D}_{\boldsymbol{R}}$, that have finite density via

$$
v_{\rho}^{N}:=\bar{v}_{\boldsymbol{\Phi}(\boldsymbol{\rho})}^{N}, \quad \rho \in \boldsymbol{R}\left(\mathcal{D}_{\boldsymbol{R}}\right)
$$

so that they are parametrised by their density. We will denote by $v_{\rho}^{\infty}:=\bigotimes_{x \in \mathbb{Z}^{d}} v_{\rho}^{1}, \rho \in$ $\boldsymbol{R}\left(\mathcal{D}_{\boldsymbol{R}}\right)$, the product measures on the configuration space $\mathbb{M}_{\infty}^{d ; 2}:=\left(\mathbb{N}_{0}^{2}\right)^{\mathbb{Z}^{d}}$ over the infinite lattice $\mathbb{Z}^{d}$. The logarithmic moment-generating function $\Lambda_{\rho}:=\Lambda_{v_{\rho}^{1}}: \mathbb{R}^{2} \rightarrow(-\infty,+\infty]$ of the one-site marginal $v_{\rho}^{1}, \rho \in \mathcal{D}_{\boldsymbol{R}}$, is defined by

$$
\Lambda_{\rho}^{1}(\lambda):=\log \int e^{\langle\lambda, \boldsymbol{k}\rangle} d v_{\rho}^{1}(\boldsymbol{k})=\log \frac{Z\left(e^{\lambda} \cdot \boldsymbol{\Phi}(\boldsymbol{\rho})\right)}{Z(\boldsymbol{\Phi}(\boldsymbol{\rho}))} .
$$

Consequently, the product and translation invariant equilibrium states have some exponential moments for all $\rho \in \boldsymbol{R}\left(\mathcal{D}_{\boldsymbol{R}}^{o}\right)$. They have full exponential moments iff $\mathcal{D}_{Z}=\mathbb{R}_{+}^{2}$.

It is easy to verify that $\boldsymbol{\Phi}(\boldsymbol{\rho})$ has a probabilistic interpretation as the one-site mean jump rate with respect to the product and translation invariant equilibrium state of density $\rho \in \boldsymbol{R}\left(\mathcal{D}_{\boldsymbol{R}}\right)$, that is

$$
\boldsymbol{\Phi}(\boldsymbol{\rho})=\int \boldsymbol{g}(\boldsymbol{\eta}(0)) d v_{\rho}^{N}, \quad \rho \in \boldsymbol{R}\left(\mathcal{D}_{\boldsymbol{R}}\right) .
$$

Since $\overline{\boldsymbol{\Phi}}=\boldsymbol{\Phi} \circ \boldsymbol{R}_{c}$, it follows by (4) that for all $\rho \in \mathbb{R}_{+}^{2}$

$$
|\overline{\boldsymbol{\Phi}}(\boldsymbol{\rho})|_{1} \leq \int|\boldsymbol{g}(\boldsymbol{\eta}(0))|_{1} d v_{\boldsymbol{R}_{c}(\boldsymbol{\rho})}^{N} \leq \boldsymbol{g}^{*} \int|\boldsymbol{\eta}(0)|{ }_{1} d v_{\boldsymbol{R}_{c}(\boldsymbol{\rho})}^{N}=\boldsymbol{g}^{*}\left|\boldsymbol{R}_{c}(\boldsymbol{\rho})\right|_{1} \leq \boldsymbol{g}^{*}|\boldsymbol{\rho}|_{1}
$$

One says that the 2-species $Z R P$ is condensing when $\boldsymbol{R}\left(\mathcal{D}_{\boldsymbol{R}}\right) \neq \mathbb{R}_{+}^{2}$, in which case there exist densities $\rho \in \mathbb{R}_{+}^{2}$ for which there is no grand canonical equilibrium state of density $\rho$. Since $\boldsymbol{R}\left(\mathcal{D}_{\boldsymbol{R}}\right)$ is non-empty and closed in $\mathbb{R}_{+}^{2}$ it follows that $\boldsymbol{R}\left(\mathcal{D}_{\boldsymbol{R}}\right) \neq \mathbb{R}_{+}^{2}$ if and only if $\partial \boldsymbol{R}\left(\mathcal{D}_{\boldsymbol{R}}\right) \neq \emptyset$, and thus condensation occurs precisely when $\mathcal{D}_{\boldsymbol{R}} \cap \partial \mathcal{D}_{\boldsymbol{R}} \neq \emptyset$. By [13, Theorem 3.3] it follows that $\boldsymbol{R}_{c}(\rho) \leq \rho$, that is, $R_{c, i}(\rho):=R_{i}(\overline{\boldsymbol{\Phi}}(\boldsymbol{\rho})) \leq \rho_{i}, i=1,2$, for all $\rho \in \mathbb{R}^{2}$. One says that condensation of the $i$-th species, $i=1$, 2, occurs at the density $\rho \in \mathbb{R}_{+}^{2}$ if $R_{c, i}(\boldsymbol{\rho})<\rho_{i}$. All cases are possible, that is, at a given density $\rho \in \mathbb{R}_{+}^{2}$ no condensation, condensation of exactly one species and condensation of both species simultaneously can occur. These cases induce an obvious partition of the phase space $\mathbb{R}_{+}^{2}$.

As proved in [13], the extension $\overline{\boldsymbol{\Phi}}$ is the correct one for the equivalence of ensembles in the sense that $\boldsymbol{R}_{c}$ gives the correct limiting background density in the thermodynamic limit. In the case of condensation, i.e., when $\boldsymbol{R}\left(\mathcal{D}_{\boldsymbol{R}}\right) \neq \mathbb{R}_{+}^{2}$, some additional assumption must be imposed on the jump rate $g$ to ensure that for each $\varphi \in \mathcal{D}_{\boldsymbol{R}} \cap \partial \mathcal{D}_{\boldsymbol{R}}$, the one-site marginal $\bar{v}_{\boldsymbol{\varphi}}^{1}$ has heavy tails in the direction normal to the set $\log \left(\mathcal{D}_{\boldsymbol{R}} \cap \partial \mathcal{D}_{\boldsymbol{R}}\right):=\left\{\log \varphi \mid \varphi \in \mathcal{D}_{\boldsymbol{R}} \cap \partial \mathcal{D}_{\boldsymbol{R}}\right\}$ at $\boldsymbol{\mu}:=\log \varphi$. Denoting by $n_{\boldsymbol{\varphi}}$ the normal to $\log \left(\mathcal{D}_{\boldsymbol{R}} \cap \partial \mathcal{D}_{\boldsymbol{R}}\right)$ at $\log \varphi\left(\right.$ where $n_{\left(\varphi_{1}, 0\right)}=\boldsymbol{e}_{1}$, $\left.n_{\left(0, \varphi_{2}\right)}=\boldsymbol{e}_{2}\right)$, this means that

$$
\lim _{\substack{\left|\boldsymbol{k}_{n}\right|_{2} \rightarrow+\infty \\ \boldsymbol{k}_{n} /\left|\boldsymbol{k}_{n}\right|_{2} \rightarrow n_{\varphi}}} \frac{1}{\left|\boldsymbol{k}_{n}\right|_{2}} \log \bar{v}_{\boldsymbol{\varphi}}^{1}\left(\boldsymbol{k}_{n}\right)=0
$$

In case $\partial \mathcal{D}_{\boldsymbol{R}}$ is not differentiable at $\boldsymbol{\varphi},(17)$ is required to hold for the two limiting normal vectors $n_{\varphi}^{+}, n_{\varphi}^{-}$at $\log \varphi$. As has been proven in [13, Lemma 3.5], a condition on the jump rate $\boldsymbol{g}$ that guarantees the critical equilibrium states have heavy tails in the direction normal to 
the logarithm of the boundary is the regularity of its tails, in the sense that for any direction $v \in S_{+}^{1}:=S^{1} \cap \mathbb{R}_{+}^{2}$,

$$
\varphi_{c ; 2}(\boldsymbol{v}):=\liminf _{\substack{|\boldsymbol{k}|_{2} \rightarrow+\infty \\ \boldsymbol{k} /|\boldsymbol{k}|_{2} \rightarrow \boldsymbol{v}}} \boldsymbol{g} !(\boldsymbol{k})^{\frac{1}{|\boldsymbol{k}|_{2}}} \in(0, \infty)
$$

exists as limit and $\varphi_{c ; 2}: S_{+}^{1} \rightarrow(0, \infty)$ is a continuous function of the direction $\boldsymbol{v} \in S_{+}^{1}$. Note that instead of the exponent $p=2$, we could have used any $p \in[1,+\infty]$, replacing the Euclidean sphere $S_{+}^{1}$ with the sphere $S_{p,+}^{1}:=\left\{\left.\boldsymbol{x} \in \mathbb{R}_{+}^{2}|| \boldsymbol{x}\right|_{p}=1\right\}$ with respect to the $\ell_{p}$-norm on $\mathbb{R}_{+}^{2}$. According to the equivalence of ensembles [13, Theorem 3.1], if the jump rate has regular tails when $\boldsymbol{R}\left(\mathcal{D}_{\boldsymbol{R}}\right) \neq \mathbb{R}_{+}^{2}$, then for all $\rho \in \mathbb{R}_{+}^{2}$

$$
\lim _{\substack{N,|\boldsymbol{K}| \rightarrow+\infty \\ \boldsymbol{K} / N^{d} \rightarrow \boldsymbol{\rho}}} \frac{1}{N^{d}} \mathcal{H}\left(v_{N, \boldsymbol{K}} \mid v_{\boldsymbol{R}_{c}(\boldsymbol{\rho})}^{N}\right)=0 .
$$

Here $\mathcal{H}(\mu \mid \nu)$ denotes the relative entropy between two probability measures $\mu, \nu$,

$$
\mathcal{H}(\mu \mid \nu):=\left\{\begin{array}{ll}
\int \frac{d \mu}{d \nu} \log \frac{d \mu}{d \nu} d v & \text { if } \mu \ll v \\
+\infty & \text { otherwise }
\end{array} .\right.
$$

The translation invariance of canonical and grand canonical ensembles and the superadditivity of the relative entropy imply convergence for any finite set $F \subseteq \mathbb{Z}^{d}$, i.e.,

$$
\lim _{\substack{N,|\boldsymbol{K}| \rightarrow+\infty \\ \boldsymbol{K} / N^{d} \rightarrow \boldsymbol{\rho}}} \mathcal{H}\left(v_{N, \boldsymbol{K}}^{F} \mid v_{\boldsymbol{R}_{c}(\boldsymbol{\rho})}^{N, F}\right)=0
$$

where $v_{N, \boldsymbol{K}}^{F}:=p_{F *} v_{N, \boldsymbol{K}}, v_{\boldsymbol{R}_{c}(\boldsymbol{\rho})}^{N, F}:=p_{F *} v_{\boldsymbol{R}_{c}(\boldsymbol{\rho})}^{N}$ are the push-forwards via the natural projection $p_{F}: \mathbb{M}_{N}^{d ; 2} \rightarrow\left(\mathbb{N}_{0}^{2}\right)^{F}$ and $\mathbb{T}_{N}^{d}$ is considered embedded in $\mathbb{Z}^{d}$. In turn this implies that $v_{N, \boldsymbol{K}}$ (considered embedded in the larger space $\mathrm{M}_{\infty}^{d ; 2}$ ) converges as $\boldsymbol{K} / N^{d} \rightarrow \rho$ to $v_{\boldsymbol{R}_{c}(\boldsymbol{\rho})}^{\infty}$ weakly with respect to bounded cylinder functions $f: \mathbb{M}_{\infty}^{d ; 2} \rightarrow \mathbb{R}$, that is, such that they depend on a finite number of coordinates.

Finally, we briefly recall the notions of local equilibrium and hydrodynamic limits and refer to [19] for more details. We say that a sequence of probability measures $\left\{\mu^{N} \in \mathbb{P}\left(\mathbb{M}_{N}^{d ; 2}\right)\right\}$ is an entropy-local equilibrium of profile $\rho \in C\left(\mathbb{T}^{d} ; \boldsymbol{R}\left(\mathcal{D}_{\boldsymbol{R}}\right)\right)$ if

$$
\limsup _{N \rightarrow+\infty} \frac{1}{N^{d}} \mathcal{H}\left(\mu^{N} \mid v_{\rho(\cdot)}^{N}\right)=0 .
$$

Here $v_{\boldsymbol{\rho}(\cdot)}^{N}:=\bigotimes_{x \in \mathbb{T}_{N}^{d}} v_{\boldsymbol{\rho}(x / N)}^{1}$ is the product measure with slowly varying parameter associated to the profile $\rho \in C\left(\mathbb{T}^{d} ; \boldsymbol{R}\left(\mathcal{D}_{\boldsymbol{R}}\right)\right)$. Given any cylinder function $f: \mathbb{M}_{N}^{d ; 2} \rightarrow \mathbb{R}$, we set $\tilde{f}(\boldsymbol{\rho}):=\int f d v_{\boldsymbol{R}_{c}(\boldsymbol{\rho})}^{N}, \boldsymbol{\rho} \in \mathbb{R}_{+}^{2}$. By a simple adaptation of [19, Corollary 6.1.3], if $\left\{\mu^{N}\right\}$ is an entropy-local equilibrium of profile $\rho \in C\left(\mathbb{T}^{d} ; \boldsymbol{R}\left(\mathcal{D}_{\boldsymbol{R}}\right)\right)$, then

$$
\lim _{N \rightarrow+\infty} \mathbb{E}_{\mu^{N}}\left|\frac{1}{N^{d}} \sum_{x \in \mathbb{T}_{N}^{d}} H\left(\frac{x}{N}\right) \tau_{x} f(\eta)-\int_{\mathbb{T}^{d}} H(u) \tilde{f}(\rho(u)) d u\right|=0
$$

for all $H \in C\left(\mathbb{T}^{d}\right)$ and all bounded cylinder functions $f: \mathbb{M}_{N}^{d ; 2} \rightarrow \mathbb{R}$, that is, $\mu^{N}$ is a weak local equilibrium of profile $\rho \in C\left(\mathbb{T}^{d} ; \boldsymbol{R}\left(\mathcal{D}_{\boldsymbol{R}}\right)\right)$. 
The hydrodynamic limit (in the diffusive timescale $t \mapsto t N^{2}$ ) of the n.n. two-species ZRP is an evolutionary PDE, such that entropy-local equilibria are conserved along its solutions (in the diffusive time-scale) in the following sense: If we start the process from an entropy local equilibrium $\mu_{0}^{N} \in \mathbb{P}\left(\mathbb{M}_{N}^{d ; 2}\right), N \in \mathbb{N}$, of some sufficiently regular initial profile $\rho_{0}: \mathbb{T}^{d} \rightarrow$ $\mathbb{R}_{+}^{2}$ at time $t=0$ and if there exists a sufficiently regular solution $\rho$ of the hydrodynamic equation on $[0, T) \times \mathbb{T}^{d}$ starting from $\rho_{0}$, then $\mu_{t}^{N}:=\mu_{0}^{N} P_{t N^{2}}^{N}$ is an entropy-local equilibrium of profile $\rho(t, \cdot)$ for each $t \in[0, T)$.

The main goal of this article is to apply the relative entropy method of H.T. Yau in order to prove the hydrodynamic limit of condensing two-species ZRPs that start from an initial entropy-local equilibrium $\left\{\mu_{0}^{N}\right\}$ of sub-critical and strictly positive profile $\rho_{0} \in$ $C\left(\mathbb{T}^{d} ; \boldsymbol{R}\left(\mathcal{D}_{\boldsymbol{R}}^{o}\right) \cap(0, \infty)^{2}\right)$, which is stated as Theorem 3.2 below. A main ingredient in the proof of the hydrodynamic limit is the one-block estimate which is stated as Theorem 3.1. The relative entropy method also requires the existence of a $C_{\mathrm{loc}}^{1,2+\theta}$ classical solution $\rho:[0, T) \times \mathbb{T}^{d} \rightarrow \boldsymbol{R}\left(\mathcal{D}_{\boldsymbol{R}}^{o}\right) \cap(0,+\infty)^{2}$ for the hydrodynamic limit and applies the Taylor expansion for $C^{2+\theta}$ functions to the function $\boldsymbol{\Phi}\left(\boldsymbol{\rho}_{t}\right)$ of the solution $\rho_{t}$ at each time $t>0$ (see (45)) in order to estimate the entropy production $\partial_{t} \mathcal{H}\left(\mu_{t}^{N} \mid v_{\boldsymbol{\rho}_{t}(\cdot)}^{N}\right)$. The sub-criticality of the solution $\rho$, i.e., that $\rho\left([0, T) \times \mathbb{T}^{d}\right) \subseteq \boldsymbol{R}\left(\mathcal{D}_{\boldsymbol{R}}^{o}\right)$, is used in Lemma 4.2 and to obtain the bound (52), which is essential in the application of Lemma 4.5. The sub-criticality of the solution $\rho$ is also required for the application of the large deviations Lemma 4.4. Together with the $C^{2+\theta}$ regularity of $\rho_{t}$ for each $t \geq 0$ it is the main assumption on the solution $\rho$. Furthermore, in the Taylor expansion the quantities $\Phi_{i}\left(\rho_{t}\right), i=1,2$, appear in the denominator, so we have to assume that the solution $\rho$ is coordinate-wise strictly positive.

As already mentioned in the introduction the expected hydrodynamic limit of the twospecies ZRP with product measures is a quasilinear parabolic system of the form (1), which in divergence form is given by

$$
\partial_{t} \rho=\operatorname{div} \mathcal{A}_{\Phi}(\rho, \nabla \rho) .
$$

Here the divergence with respect to the spatial parameter is applied coordinate-wise, and $\nabla \rho(t, u):=\left(\nabla \rho_{1}(t, u), \nabla \rho_{2}(t, u)\right) \in \mathbb{R}^{2 \times d}$ is the gradient of $\rho$ with respect to the spatial variable $u \in \mathbb{T}^{d}$. Furthermore, $\mathcal{A}_{\boldsymbol{\Phi}}=\left(A_{\boldsymbol{\Phi}}^{1}, \mathcal{A}_{\boldsymbol{\Phi}}^{2}\right): \boldsymbol{R}\left(\mathcal{D}_{\boldsymbol{R}}^{o}\right) \times \mathbb{R}^{2 \times d} \rightarrow \mathbb{R}^{2 \times d}$ is the function given by

$$
\mathcal{A}_{\Phi}(\rho, V)=D \Phi(\rho) V
$$

that is,

$$
\partial_{t} \rho_{i}=\operatorname{div} \mathcal{A}_{\Phi}^{i}(\rho, \nabla \rho)=\operatorname{div}\left(\nabla \Phi_{i}(\boldsymbol{\rho}) \nabla \boldsymbol{\rho}\right)=\Delta \Phi_{i}(\boldsymbol{\rho}), \quad i=1,2 .
$$

Structural properties of the mobility matrix $D \boldsymbol{\Phi}: \boldsymbol{R}\left(\mathcal{D}_{\boldsymbol{R}}^{o}\right) \rightarrow \mathbb{R}^{2 \times 2}$ can be inferred by the properties of $D \boldsymbol{R}$. For example, for all $\rho \in \boldsymbol{R}\left(\mathcal{D}_{\boldsymbol{R}}^{o}\right) \cap(0,+\infty)^{2}$,

$$
D \boldsymbol{\Phi}(\boldsymbol{\rho})=\left(\begin{array}{ll}
\Phi_{1}(\boldsymbol{\rho}) & 0 \\
0 & \Phi_{2}(\boldsymbol{\rho})
\end{array}\right) D^{2} S(\boldsymbol{\rho}),
$$

where $D^{2} S(\rho)=D(\log \boldsymbol{\Phi})(\boldsymbol{\rho})$ is a strictly positive definite matrix, the second derivative of the thermodynamic entropy, and for all $\rho \in \boldsymbol{R}\left(\mathcal{D}_{\boldsymbol{R}}^{o}\right)$, the relations (13) hold and

$$
\partial_{1} \Phi_{1}(\rho) \wedge \partial_{2} \Phi_{2}(\rho)>0 .
$$

In particular, $D \boldsymbol{\Phi}(\rho)$ has positive eigenvalues for all $\rho \in \boldsymbol{R}\left(\mathcal{D}_{\boldsymbol{R}}^{o}\right)$ and is diagonisable for all $\rho \in \boldsymbol{R}\left(\mathcal{D}_{\boldsymbol{R}}^{o}\right) \cap(0,+\infty)^{2}$. For $\rho \in \boldsymbol{R}\left(\mathcal{D}_{\boldsymbol{R}}^{o}\right)$ with $\rho_{1} \rho_{2}=0$, the matrix $D \boldsymbol{\Phi}(\boldsymbol{\rho})$ is triangular. 
It follows that although $D \Phi(\rho)$ is not necessarily symmetric, it is uniformly parabolic away from the critical densities, that is, for any compact $K \subseteq \boldsymbol{R}\left(\mathcal{D}_{\boldsymbol{R}}^{o}\right)$ the exists $\lambda_{K}>0$ such that

$$
\langle\boldsymbol{\xi}, D \boldsymbol{\Phi}(\boldsymbol{\rho}) \boldsymbol{\xi}\rangle \geq \lambda_{K}|\boldsymbol{\xi}|^{2}, \quad \rho \in K, \boldsymbol{\xi} \in \mathbb{R}^{2} .
$$

By the work [1] of Amann, it is known that for $C^{2+\theta}$ initial data, uniformly parabolic systems in general form have unique maximal $C^{1,2+\theta}$ solutions. Thus, since $D \boldsymbol{\Phi}$ is uniformly parabolic in any compact subset of $\boldsymbol{R}\left(\mathcal{D}_{\boldsymbol{R}}^{o}\right)$, it follows that for initial data $\boldsymbol{\rho}_{0} \in$ $C^{2+\theta}\left(\mathbb{T}^{d} ; \boldsymbol{R}\left(\mathcal{D}_{\boldsymbol{R}}^{o}\right) \cap(0,+\infty)^{2}\right)$ there exists a unique maximal classical $C_{\text {loc }}^{1,2+\theta}$ solution $\rho:\left[0, T_{\max }\right) \times \mathbb{T}^{d} \rightarrow \boldsymbol{R}\left(\mathcal{D}_{\boldsymbol{R}}^{o}\right)$ of the parabolic system (1) taking values in the sub-critical region $\boldsymbol{R}\left(\mathcal{D}_{\boldsymbol{R}}^{o}\right)$. Of course, since we also assume $\boldsymbol{\rho}_{0}\left(\mathbb{T}^{d}\right) \subseteq(0,+\infty)^{2}$, by taking if necessary $T_{\max }$ to be smaller we can assume that $\rho$ takes values in $\boldsymbol{R}\left(\mathcal{D}_{\boldsymbol{R}}^{o}\right) \cap(0,+\infty)^{2}$. This establishes the local in time existence of $C^{1,2+\theta}$ sub-critical solutions $\rho$. On the other hand, by the regularity theory of quasilinear uniformly parabolic systems of the form (22), see [5, Theorem 1.2] and the references therein, it is known that weak solutions to such systems exhibit singularities on a closed subset $Q \subseteq[0, T] \times \mathbb{T}^{d}$ of zero measure. So we can not simply apply the $C^{2+\theta}$ Taylor expansion on the function $\Phi\left(\rho_{t}\right)$ for all times $t \geq 0$. Furthermore we do not know whether the sub-critical region $\boldsymbol{R}\left(\mathcal{D}_{\boldsymbol{R}}\right)$ is an invariant region for the zero range parabolic system (22). These are the two main reasons that force us to rely on Amann's local in time existence of regular solutions, and prove a local in time version of the hydrodynamic limit. A further study of the PDE system arising as the hydrodynamic limit of a two-species ZRP, although interesting, is outside of the scope of this article, which is the passage from the microscopic to the macroscopic description.

However, in the example of the species-blind ZRP one can take into advantage its relation

with a particular one-species ZRP to obtain the global in time existence of $C_{\text {loc }}^{1+\theta, 2+\theta}$ solutions and a type of maximum principle, in which the sub-critical region plays the role of the invariant domain. We prove this in Theorem 3.3.

\subsection{The Species-Blind ZRP}

We now consider two-species local jump rate functions of the form

$$
g_{1}(\boldsymbol{k})=k_{1} h\left(k_{1}+k_{2}\right), \quad g_{2}(\boldsymbol{k})=k_{2} h\left(k_{1}+k_{2}\right)
$$

for some function $h: \mathbb{N}_{0} \rightarrow \mathbb{R}_{+}$satisfying the non-degeneracy condition $h(k)>0$ for all $k \in \mathbb{N}$. Any jump rate $g$ of this form satisfies (6) since

$$
g_{1}(\boldsymbol{k}) g_{2}\left(\boldsymbol{k}-\boldsymbol{e}_{1}\right)=k_{1} h\left(k_{1}+k_{2}\right) k_{2} h\left(k_{1}+k_{2}-1\right)=g_{1}\left(\boldsymbol{k}-\boldsymbol{e}_{2}\right) g_{2}(\boldsymbol{k})
$$

for all $\boldsymbol{k} \in \mathbb{N}^{2}$ and the factorial of such a jump rate is given by

$$
\boldsymbol{g} !(\boldsymbol{k})=1 \cdot h(1) \cdot \ldots \cdot k_{1} \cdot h\left(k_{1}\right) \cdot 1 \cdot h\left(k_{1}+1\right) \cdot \ldots \cdot k_{2} \cdot h\left(k_{1}+k_{2}\right)=k_{1} ! k_{2} ! h !\left(k_{1}+k_{2}\right) .
$$

The partition function associated to $\boldsymbol{g}$ is given for $\boldsymbol{\varphi} \in \mathbb{N}_{0}^{2}$ with $\varphi_{2}>0$ by

$$
Z(\boldsymbol{\varphi})=\sum_{m=0}^{\infty} \frac{\varphi_{2}^{m}}{h !(m)} \sum_{k_{1}=0}^{m} \frac{\left(\frac{\varphi_{1}}{\varphi_{2}}\right)^{k_{1}}}{k_{1} !\left(m-k_{1}\right) !}=\sum_{m=0}^{\infty} \frac{\varphi_{2}^{m}}{m ! h !(m)}\left(1+\frac{\varphi_{1}}{\varphi_{2}}\right)^{m}=\hat{Z}\left(\varphi_{1}+\varphi_{2}\right),
$$

where $\hat{Z}$ is the partition function associated to the one-species rate function $\hat{g}(k):=k h(k)$. So, in what follows, we assume that $h$ is of the form $h(k)=\frac{\hat{g}(k)}{k}, k \geq 1$, for some onespecies local jump rate function $\hat{g}$ with regular tails, i.e., such that the limit inferior $\hat{\varphi}_{c}:=$ 
$\lim \inf _{k \rightarrow+\infty} \hat{g} !(k)^{\frac{1}{k}}>0$ exists as a limit. In this case the function $\boldsymbol{g}$ defined in (23) is a two-species local jump rate. Indeed, the non-degeneracy condition (2) and the Lipschitz condition (3) are easy to verify, as we have seen $\boldsymbol{g}$ satisfies the compatibility condition (6) and obviously $\mathcal{D}_{Z}=\left\{\boldsymbol{\varphi} \in \mathbb{R}_{+}^{2} \mid \varphi_{1}+\varphi_{2} \in \mathcal{D}_{\hat{Z}}\right\}$ and $\mathcal{D}_{\boldsymbol{R}}=\left\{\boldsymbol{\varphi} \in \mathbb{R}_{+}^{2} \mid \varphi_{1}+\varphi_{2} \in \mathcal{D}_{\hat{R}}\right\}$, where $\hat{R}(\varphi)=\varphi(\log \hat{Z})^{\prime}(\varphi)$ is the density function associated to the one-species jump rate $\hat{g}$. In particular $\mathcal{D}_{Z} \neq \varnothing$ and thus also (8) holds. We will refer to this nearest neighbour two-species ZRP as the species-blind ZRP corresponding to the 1-species jump rate $\hat{g}$. The density function corresponding to $g$ is given by the formula

$$
\boldsymbol{R}(\boldsymbol{\varphi})=\left(\frac{\varphi_{1} \hat{Z}^{\prime}\left(\varphi_{1}+\varphi_{2}\right)}{\hat{Z}\left(\varphi_{1}+\varphi_{2}\right)}, \frac{\varphi_{2} \hat{Z}^{\prime}\left(\varphi_{1}+\varphi_{2}\right)}{\hat{Z}\left(\varphi_{1}+\varphi_{2}\right)}\right)=\frac{\hat{R}\left(|\varphi|_{1}\right)}{|\varphi|_{1}} \boldsymbol{\varphi} .
$$

We set $\hat{\Phi}:=\hat{R}^{-1}$ and we will compute the inverse $\boldsymbol{\Phi}$ of $\boldsymbol{R}: \mathcal{D}_{\boldsymbol{R}} \rightarrow \mathbb{R}_{+}^{2}$ in its image $\boldsymbol{R}\left(\mathcal{D}_{\boldsymbol{R}}\right)$. Let $\rho=\boldsymbol{R}(\varphi)$. We have to solve the system

$$
\rho_{1}=\frac{\varphi_{1} \hat{Z}^{\prime}\left(\varphi_{1}+\varphi_{2}\right)}{\hat{Z}\left(\varphi_{1}+\varphi_{2}\right)}, \quad \rho_{2}=\frac{\varphi_{2} \hat{Z}^{\prime}\left(\varphi_{1}+\varphi_{2}\right)}{\hat{Z}\left(\varphi_{1}+\varphi_{2}\right)}
$$

for $\left(\varphi_{1}, \varphi_{2}\right)$. By adding the two equations we obtain that $\rho_{1}+\rho_{2}=\hat{R}\left(\varphi_{1}+\varphi_{2}\right)$. In particular $\rho_{1}+\rho_{2} \in \hat{R}\left(\mathcal{D}_{\hat{R}}\right)$ for all $\rho \in \boldsymbol{R}\left(\mathcal{D}_{\boldsymbol{R}}\right)$ and $\varphi_{1}+\varphi_{2}=\hat{\Phi}\left(\rho_{1}+\rho_{2}\right)$. Substituting $\varphi_{1}+\varphi_{2}$ with $\hat{\Phi}\left(\rho_{1}+\rho_{2}\right)$ in both equations in $(24)$, we can solve for $\left(\varphi_{1}, \varphi_{2}\right)$ to obtain

$$
\varphi_{i}=\rho_{i} \frac{\hat{Z}\left(\hat{\Phi}\left(\rho_{1}+\rho_{2}\right)\right)}{\hat{Z}^{\prime}\left(\hat{\Phi}\left(\rho_{1}+\rho_{2}\right)\right)}=\rho_{i} \frac{1}{\left(\log \hat{Z}^{\prime}\left(\hat{\Phi}\left(\rho_{1}+\rho_{2}\right)\right)\right.}=\rho_{i} \frac{\hat{\Phi}\left(\rho_{1}+\rho_{2}\right)}{\rho_{1}+\rho_{2}},
$$

where the last equality above follows from the identity $\hat{R}(\varphi)=\varphi(\log Z)^{\prime}(\varphi)$ for the onespecies density and partition functions, since by this identity we have for all $\rho \in\left(0, \hat{\rho}_{c}\right)$ that

$$
\frac{1}{(\log \hat{Z})^{\prime}(\hat{\Phi}(\rho))}=\frac{\hat{\Phi}(\rho)}{\hat{R}(\hat{\Phi}(\rho))}=\frac{\hat{\Phi}(\rho)}{\rho},
$$

where $\hat{\rho}_{c}$ is the corresponding critical density of the one-species jump rate $\hat{g}$. Consequently, the inverse $\boldsymbol{\Phi}:=\boldsymbol{R}^{-1}: \boldsymbol{R}\left(\mathcal{D}_{\boldsymbol{R}}\right) \rightarrow \mathcal{D}_{\boldsymbol{R}}$ is given by the formula

$$
\boldsymbol{\Phi}(\boldsymbol{\rho})=\left(\rho_{1} \frac{\hat{\Phi}\left(\rho_{1}+\rho_{2}\right)}{\rho_{1}+\rho_{2}}, \rho_{2} \frac{\hat{\Phi}\left(\rho_{1}+\rho_{2}\right)}{\rho_{1}+\rho_{2}}\right)=\frac{\hat{\Phi}\left(|\boldsymbol{\rho}|_{1}\right)}{|\boldsymbol{\rho}|_{1}} \boldsymbol{\rho} .
$$

Thus the expected hydrodynamic equation of the species-blind ZRP is

$$
\partial_{t} \rho_{i}=\Delta\left(\rho_{i} \frac{\hat{\Phi}\left(\rho_{1}+\rho_{2}\right)}{\rho_{1}+\rho_{2}}\right), \quad i=1,2 .
$$

Since (26) is the expected hydrodynamic equation of the species-blind ZRP we will refer to it as the species-blind parabolic system. A classical solution to the species-blind parabolic system is a $C^{1,2}$ function $\rho=\left(\rho_{1}, \rho_{2}\right):[0, T) \times \mathbb{T}^{d} \rightarrow \mathbb{R}^{2}$ satisfying (26) with $0 \leq$ $\rho_{1}(t, u)+\rho_{2}(t, u)<\hat{\rho}_{c}$ for all $(t, u) \in[0, T) \times \mathbb{T}^{d}$. Note that for any classical solution $\rho=\left(\rho_{1}, \rho_{2}\right)$ of the species-blind parabolic system (26) the sum $\rho_{1}+\rho_{2}$ satisfies the parabolic equation $\partial_{t} \rho=\hat{\Phi}(\rho)$ corresponding to the 1 -species ZRP of jump rate $\hat{g}(k)=k h(k)$. This remark will allows us to prove the global in time existence of solutions to the species-blind parabolic system. A similar argument was used for two-species simple exclusion processes in [21]. 
As an example of the nice properties of the species-blind process, we note that the extended mean jump rate $\overline{\boldsymbol{\Phi}}: \mathbb{R}_{+}^{2} \rightarrow \mathcal{D}_{\boldsymbol{R}}$ of the species-blind process can be computed explicitly and is given by

$$
\bar{\Phi}(\rho)=\frac{\overline{\hat{\Phi}}\left(|\rho|_{1}\right)}{|\rho|_{1}} \rho,
$$

where $\overline{\hat{\Phi}}(\rho)=\hat{\Phi}\left(\rho \wedge \hat{\rho}_{c}\right), \rho \geq 0$, is the extended mean jump rate of the one-species ZRP with jump rate $\hat{g}$.

\section{Main Results}

A main probabilistic ingredient in the proof of the hydrodynamic limit of ZRPs is the so-called one-block estimate, which is well known under assumptions that exclude condensing ZRPs (e.g., [19, Sect. 5.4]). Our first result is a version of the one-block estimate for condensing ZRPs, i.e., $\boldsymbol{R}\left(\mathcal{D}_{\boldsymbol{R}}\right) \neq \mathbb{R}_{+}^{2}$, under the additional assumptions that the local jump rate $\boldsymbol{g}$ is bounded, has a continuous partition function $Z$, and has regular tails in the sense of (18). We note that these extra assumptions in the one-block estimate and the hydrodynamic limit below are not required in the non-condensing case, i.e., when $\boldsymbol{R}\left(\mathcal{D}_{\boldsymbol{R}}\right)=\mathbb{R}_{+}^{2}$. In the case that $\boldsymbol{R}\left(\mathcal{D}_{\boldsymbol{R}}\right)=\mathbb{R}_{+}^{2}$, but $\mathcal{D}_{Z} \neq \mathbb{R}_{+}^{2}$, Theorems 3.1 and 3.2 still hold under the (weaker than boundedness) assumption that $g$ has sub-linear growth at infinity in the sense that

$$
\limsup _{|\boldsymbol{k}|_{1} \rightarrow+\infty} \frac{|\boldsymbol{g}(\boldsymbol{k})|_{1}}{|\boldsymbol{k}|_{1}}=0
$$

In the case that $\boldsymbol{R}\left(\mathcal{D}_{\boldsymbol{R}}\right)=\mathcal{D}_{Z}=\mathbb{R}_{+}^{2}$, no extra assumption is required on $\boldsymbol{g}$. Given any (cylinder) function $f: \mathbb{M}_{N}^{d ; 2} \rightarrow \mathbb{R}^{2}$ we set

$$
f^{\ell}:=\frac{1}{(2 \ell+1)^{d}} \sum_{|x| \leq \ell} \tau_{x} f
$$

where $\tau_{x} \boldsymbol{f}(\boldsymbol{\eta}):=\boldsymbol{f}\left(\tau_{x} \boldsymbol{\eta}\right)$ and $\tau_{x} \boldsymbol{\eta}(y):=\boldsymbol{\eta}(x+y)$ for $x, y \in \mathbb{T}_{N}^{d}$.

Theorem 3.1 (One-block estimate) Suppose that the ZRP is condensing and that the local jump rate $\mathbf{g}$ of the $Z R P$ is bounded, has regular tails in the sense of (18) and its partition function $Z$ is continuous on $\mathcal{D}_{Z} \cap \partial \mathcal{D}_{Z}$. Then for any sequence of initial distributions $\mu_{0}^{N} \in$ $\mathbb{P}\left(\mathbb{M}_{N}^{d ; 2}\right)$ satisfying the $O\left(N^{d}\right)$-entropy assumption, i.e.,

$$
C(\boldsymbol{a}):=\limsup _{N \in \mathbb{N}} \frac{1}{N^{d}} \mathcal{H}\left(\mu_{0}^{N} \mid v_{\boldsymbol{a}}^{N}\right)<+\infty,
$$

for some (and thus for any) $\boldsymbol{a} \in \boldsymbol{R}\left(\mathcal{D}_{\boldsymbol{R}}^{o}\right) \cap(0, \infty)^{2}$, it holds that

$$
\lim _{\ell \rightarrow \infty} \limsup _{N \rightarrow \infty} \mathbb{E}^{N}\left|\int_{0}^{T} \frac{1}{N^{d}} \sum_{x \in \mathbb{T}_{N}^{d}}\left\langle\boldsymbol{F}\left(t, \frac{x}{N}\right), \boldsymbol{g}\left(\boldsymbol{\eta}_{t}(x)\right)-\overline{\boldsymbol{\Phi}}\left(\boldsymbol{\eta}_{t}(x)^{\ell}\right)\right\rangle d t\right|=0
$$

for all functions $\boldsymbol{F} \in C\left([0, T] \times \mathbb{T}^{d} ; \mathbb{R}^{2}\right), T>0 ; \mathbb{E}^{N}$ denotes the expectation with respect to the diffusively accelerated law of the $Z R P$ starting from $\mu_{0}^{N} \in \mathbb{P}\left(\mathbb{M}_{N}^{d ; 2}\right)$ and $\overline{\boldsymbol{\Phi}}$ is the extension of $\boldsymbol{\Phi}$ given by (11).

We note that the extension $\overline{\boldsymbol{\Phi}}$ of the mean jump rate is required in the statement of the one-block estimate, because $\eta_{t}^{\ell}$ can be outside the domain of sub-critical densities. This is 
the correct extension due to the equivalence of ensembles. The proof of this result is given in Sect. 4.1 below.

Next is the general result regarding the hydrodynamic limit of two-species ZRPs. As noted in the introduction, in order to take into account condensing ZRPs, we apply the relative entropy method of H.T. Yau which requires only the one-block estimate and not the full replacement lemma. But this method relies on the existence of sufficiently regular classical solutions of parabolic systems which are known to exist only locally in time, and thus the result is local in time, valid for the time interval that the unique maximal classical solution of (1) established in [1] exists. We denote by $C^{1+a, 2+b}\left([0, T] \times \mathbb{T}^{d}\right), a, b \in[0,1)$, the space of all $C^{1,2}$-functions $f:[0, T] \times \mathbb{T}^{d} \rightarrow \mathbb{R}$ such that $\partial_{t} f \in C^{a}\left([0, T] \times \mathbb{T}^{d}\right)$ is $a$-Hölder continuous and $\partial_{i j}^{2} f \in C^{b}\left([0, T] \times \mathbb{T}^{d}\right)$ is $b$-Hölder continuous, where $[0, T] \times \mathbb{T}^{d}$ is equipped with the parabolic metric $d$ given by

$$
d((t, x),(s, y))=\left(d_{\mathbb{T}^{d}}(x, y)^{2}+|t-s|\right)^{\frac{1}{2}} .
$$

As usual, if $I \subseteq \mathbb{R}$ is an interval, then we write $C_{\text {loc }}^{1+a, 2+b}\left(I \times \mathbb{T}^{d}\right)$ for the space of all functions $f$ such that $f \in C^{1+a, 2+b}\left(J \times \mathbb{T}^{d}\right)$ for any compact sub-interval $J \subseteq I$. This is extended coordinate-wise to vector-valued functions; given a subset $A \subseteq \mathbb{R}^{2}$, we denote by $C_{\mathrm{loc}}^{1+a, 2+b}\left(I \times \mathbb{T}^{d} ; A\right)$ the subset of $C_{\mathrm{loc}}^{1+a, 2+b}\left(I \times \mathbb{T}^{d} ; \mathbb{R}^{2}\right)$ consisting of functions taking values in $A$.

Theorem 3.2 (Hydrodynamic limit) Let $\left(S_{t}^{N}\right)_{t \geq 0}$ be the transition semigroup of the twospecies symmetric n.n. ZRP on the torus $\mathbb{T}_{N}^{d}, N \in \mathbb{N}$, with condensing jump rate $g$ satisfying the assumptions of the one-block estimate above, and let $\boldsymbol{\Phi}$ be the mean jump rate associated to $\mathrm{g}$. Let

$$
\rho \in C_{\mathrm{loc}}^{1,2+\theta}\left(\left[0, T_{\max }\right) \times \mathbb{T}^{d} ; \boldsymbol{R}\left(\mathcal{D}_{\boldsymbol{R}}^{o}\right) \cap(0, \infty)^{2}\right)
$$

be the unique maximal solution of the parabolic system (1) with values in the sub-critical region $\boldsymbol{R}\left(\mathcal{D}_{\boldsymbol{R}}^{o}\right) \cap(0,+\infty)^{2}$ of strictly positive densities. Then any initial entropy local equilibrium $\mu_{0}^{N} \in \mathbb{P}\left(\mathbb{M}_{N}^{d ; 2}\right)$ is conserved along the solution $\rho$. In other words, if $\left\{\mu_{0}^{N}\right\}$ is an entropy-local equilibrium of profile $\rho_{0}:=\rho(0, \cdot) \in C^{2+\theta}\left(\mathbb{T}^{d}\right)$ then $\mu_{t}^{N}:=S_{t N^{2}}^{N} \mu_{0}^{N}$, with $N \in \mathbb{N}$, is an entropy local equilibrium for all $t \in\left[0, T_{\max }\right)$. In particular, $\left\{\mu_{t}^{N}\right\}$ satisfies (21) for all $t \in\left[0, T_{\max }\right)$.

This theorem is proved in Sect. 4.2. We should note that, although the proof of the hydrodynamic limit relies strongly on the assumption that the classical solution $\rho$ takes values in the set $\boldsymbol{R}\left(\mathcal{D}_{\boldsymbol{R}}^{o}\right) \cap(0, \infty)^{2}$ for all times $t \geq 0$, and so in particular requires the sequence of initial distributions $\left\{\mu_{0}^{N}\right\}$ to be an entropy local equilibrium of some sub-critical and strictly positive profile $\rho_{0} \equiv \rho(0, \cdot)$, the one-block estimate does not require this assumption. It only requires that $\left\{\mu_{0}^{N}\right\}$ satisfies the $O\left(N^{d}\right)$-entropy assumption, which can hold even for super-critical profiles, having a Dirac mass of order $O\left(N^{d}\right)$ at some site $x \in \mathbb{T}^{d}$, e.g., $\mu_{0}^{N}(d \boldsymbol{\eta})=\delta_{\left[\boldsymbol{a} N^{d}\right]}\left(d \boldsymbol{\eta}_{[N x]}\right) \otimes \bigotimes_{y \neq[N x]} v_{\boldsymbol{\rho}(y / N)}^{1}\left(d \boldsymbol{\eta}_{y}\right)$ with $\boldsymbol{a} \in(0, \infty)^{2}$, when $\boldsymbol{R}\left(\mathcal{D}_{\boldsymbol{R}}\right) \neq \mathbb{R}_{+}^{2}$.

We note also that the assumption that $\rho\left(\left[0, T_{\max }\right) \times \mathbb{T}^{d}\right) \subseteq(0,+\infty)^{2}$ is a technical one, arising from the fact the $\boldsymbol{\Phi}\left(\rho_{t}\right)$ appears in the denominator. If one knew that the region $\mathbb{R}_{+}^{2}$ is strongly invariant for the parabolic system (1) in the sense that $\rho_{1} \wedge \rho_{2}$ becomes strictly positive (and sufficiently fast) for the solution $\rho$, then one can replace the assumption $\rho\left(\left[0, T_{\max }\right) \times \mathbb{T}^{d}\right) \subseteq(0,+\infty)^{2}$ with the assumption $\rho_{0} \geq 0$ as in [23, Remark 3.3] for the one-species case. Secondly, if one knew that the region $(0,+\infty)^{2}$ is invariant for the parabolic system (1), then starting from $C^{2+\theta}$ non-negative initial data $\rho_{0}: \mathbb{T}^{d} \rightarrow \boldsymbol{R}\left(\mathcal{D}_{\boldsymbol{R}}^{o}\right)$ 
one could could choose small enough $\varepsilon>0$ such that $\boldsymbol{\rho}_{0}^{\varepsilon}\left(\mathbb{T}^{d}\right) \subseteq \boldsymbol{R}\left(\mathcal{D}_{\boldsymbol{R}}^{o}\right) \cap(0,+\infty)^{2}$ where $\rho_{0, i}^{\varepsilon}=\rho_{0, i}+\varepsilon, i=1,2$, use the result for strictly positive data and try to pass to the limit as $\varepsilon \rightarrow 0$. Since we do not pursue the study of the quasilinear parabolic system (1) and its invariant regions at the macroscopic level in this article, we consider only local solutions which are strictly positive and sub-critical and whose existence is established by Amann [1].

The next result states that, when starting from sufficiently regular subcritical initial profiles, the species-blind system (26) has solutions defined globally in time.

Theorem 3.3 (Global existence for the species-blind parabolic system) Let $\rho_{0} \in C^{2+\theta}\left(\mathbb{T}^{d}\right.$; $\left.\boldsymbol{R}\left(\mathcal{D}_{\boldsymbol{R}}^{o}\right) \cap(0, \infty)^{2}\right), \theta \in[0,1)$, be an initial profile. Then the species-blind parabolic system (26) has a unique classical solution $\rho: \mathbb{R}_{+} \times \mathbb{T}^{d} \rightarrow \mathbb{R}^{2}$ starting from $\boldsymbol{\rho}_{0}$ and

$$
\rho \in C_{\mathrm{loc}}^{1+\theta, 2+\theta}\left([0,+\infty) \times \mathbb{T}^{d} ; \boldsymbol{R}\left(\mathcal{D}_{\boldsymbol{R}}^{o}\right) \cap(0, \infty)^{2}\right) .
$$

The proof of this Theorem can be found in Subsect. 4.3 and it is obtained by taking into account the fact that the sum $\rho_{1}+\rho_{2}$ of the two variables of a solution $\rho=\left(\rho_{1}, \rho_{2}\right)$ of the species-blind parabolic system is a solution of the scalar parabolic equation $\partial_{t}(\rho)=\Delta \hat{\Phi}(\rho)$. Here, by using the strong maximum principle for scalar quasilinear parabolic equations and by proving that classical solutions $\rho$ of the species-blind parabolic system do not become negative, we obtain that the the sub-critical region is an invariant region. We believe that $\mathbb{R}_{+}^{2}$ will be an invariant region of the species-blind parabolic system in general. Yet, since we do not study this question in this article, in order to be rigorous we prove it in this particular case. We should add that the arguments used strongly rely on the relation to the PDE of the single species ZRP associated to the species-blind ZRP by "ignoring" the species, and thus do not easily extend to the general case.

As a corollary, we find that the hydrodynamic limit for the species-blind process holds globally in time; Subsect. 4.4 gives the proof.

Corollary 3.1 Let $\left(S_{t}^{N}\right)_{t \geq 0}$ be the transition semigroup of the diffusively rescaled speciesblind symmetric n.n. ZRP on the torus $\mathbb{T}_{N}^{d}$ corresponding to a one-species jump rate $\hat{g}$ such that $\hat{\varphi}_{c}:=\liminf _{k \rightarrow+\infty} \hat{g} !(k)^{\frac{1}{k}} \in(0,+\infty]$ exists as a limit. Assume further that $\hat{g}$ is bounded if the critical density $\hat{\rho}_{c}$ of the one-species $Z R P$ is finite. If $\mu_{0}^{N} \in \mathbb{P}\left(\mathbb{M}_{N}^{d ; 2}\right)$ is an entropy local equilibrium of profile $\rho_{0} \in C^{2+\theta}\left(\mathbb{T}^{d} ; \boldsymbol{R}\left(\mathcal{D}_{\boldsymbol{R}}^{o}\right) \cap(0, \infty)^{2}\right)$, then $\mu_{t}^{N}:=\mu_{0}^{N} S_{t}^{N}$ is an entropy local equilibrium of profile $\rho(t, \cdot)$ for all $t \geq 0$, where $\rho \in C_{\mathrm{loc}}^{1+\theta, 2+\theta}\left(\mathbb{R}_{+} \times \mathbb{T}^{d} ; \boldsymbol{R}\left(\mathcal{D}_{\boldsymbol{R}}^{o}\right) \cap\right.$ $\left.(0, \infty)^{2}\right)$ is the unique solution to the species-blind parabolic system (26) starting from $\boldsymbol{\rho}_{0}$.

\section{Proofs}

\subsection{Proof of Theorem 3.1}

The proof of the one-block estimate follows closely the proof for the one-species case found in [19, Sect. 5.4]. The differences are twofold. In [19, Sect. 5.4], the one-species case is treated, and we extend this result to two species. However, the main difference is that in [19] the non-condensing case is treated, while we cover the condensing case as well. This is shown by applying the equivalence of ensembles (19) as in [23].

The first step in the proof of the one-block estimate is to replace the jump rate $g(\eta(x))$ at the site $x$ with the spatial average $\boldsymbol{g}(\boldsymbol{\eta}(x))^{\ell}$ over a box of size $\ell \in \mathbb{N}_{0}$. This is based on the following lemma which is also useful in the proof of Theorem 3.2. The proof is omitted as it is a simple adaptation of the proof for the one-species case [19, Lemma 6.4.1]. 
Lemma 4.1 If the sequence $\left\{\mu_{0}^{N}\right\}$ of initial distributions satisfies the $O\left(N^{d}\right)$-entropy assumption (28), then

$$
\int|\eta|_{1} d \mu_{0}^{N} \leq O\left(N^{d}\right)
$$

where $|\boldsymbol{\eta}|_{1}:=|\boldsymbol{\eta}|_{N, 1}:=\sum_{x \in \mathbb{T}_{N}^{d}}|\boldsymbol{\eta}(x)|_{1}$.

This lemma, a change of variables and the conservation of the number of particles allow us to replace $\boldsymbol{g}(\boldsymbol{\eta}(x))$ with the spatial average $\boldsymbol{g}(\boldsymbol{\eta}(x))^{\ell}$ in the statement of the one-block estimate, and thus the one-block estimate is reduced to proving that

$$
\lim _{\ell \rightarrow \infty} \limsup _{N \rightarrow \infty} \int \frac{1}{N^{d}} \sum_{x \in \mathbb{T}^{d}} \tau_{x} V^{\ell} d \bar{\mu}_{T}^{N}=0
$$

where $\bar{\mu}_{T}^{N}:=\frac{1}{T} \int_{0}^{T} \mu_{t}^{N} d t$ and $V^{\ell}$ is the cylinder function $V^{\ell}:=\left|\boldsymbol{g}(\boldsymbol{\eta}(0))^{\ell}-\overline{\boldsymbol{\Phi}}\left(\boldsymbol{\eta}(0)^{\ell}\right)\right|_{1}$.

We establish this identity in a sequence of steps. We first estimate the entropy and the Dirichlet form of the density $\bar{f}_{T}^{N}:=d \bar{\mu}_{T}^{N} / d \nu_{\rho_{\star}}^{N}$ of $\bar{\mu}_{T}^{N}$ with respect to an equilibrium state of density $\boldsymbol{\rho}_{*} \in A$. Note that $\bar{f}_{T}^{N}=\frac{1}{T} \int f_{t}^{N} d t$, where $f_{t}^{N}:=d \mu_{t}^{N} / d v_{\boldsymbol{\rho}_{*}}^{N}$ is the density of the law $\mu_{t}^{N}$ of the ZRP at time $t$ with respect to the product equilibrium state of density $\boldsymbol{\rho}_{*} \in A$. By [19, Proposition A.9.1], for any initial probability measure $\mu$ the entropy $H\left(\mu_{t} \mid v\right)$ of the law $\mu_{t}:=\mu P_{t}$ of a Markov semigroup $\left(P_{t}\right)_{t \geq 0}$ at time $t$ with respect to an equilibrium state $\pi$ of $\left(P_{t}\right)$ is a non-increasing function of time. Here the equilibrium $\pi$ need not be unique or approached by $\mu_{t}$ as $t \rightarrow+\infty$. Therefore, since $\mu_{0}^{N}$ satisfies the $O\left(N^{d}\right)$-entropy assumption, we have for fixed $\boldsymbol{\rho}_{*} \in A$ that $H\left(\mu_{t}^{N} \mid v_{\boldsymbol{\rho}_{*}}^{N}\right) \leq C\left(\boldsymbol{\rho}_{*}\right) N^{d}$, which, by convexity of the entropy, implies that $H\left(\bar{\mu}_{T}^{N} \mid v_{\boldsymbol{\rho}_{*}}^{N}\right) \leq C\left(\boldsymbol{\rho}_{*}\right) N^{d}$. Furthermore, if $D_{N}: L_{+}^{1}\left(v_{\boldsymbol{\rho}_{*}}^{N}\right) \rightarrow[0,+\infty]$ denotes the functional defined by $D_{N}(f)=\mathfrak{D}_{N}(\sqrt{f})$ where $\mathfrak{D}_{N}: L^{2}\left(v_{\boldsymbol{\rho}_{*}}\right) \rightarrow[0,+\infty]$ is the Dirichlet form associated to the generator $L_{N}$,

$$
\mathfrak{D}_{N}(f):=-\left\langle f, L_{N} f\right\rangle_{\nu_{\rho_{*}}}=-\int f L_{N} f d v_{\boldsymbol{\rho}_{*}},
$$

then by [19, Proposition A.9.2] and the convexity of the functional $D_{N}$, it follows that $D_{N}\left(\bar{f}_{T}^{N}\right) \leq \frac{1}{T} \int_{0}^{T} D_{N}\left(f_{t}^{N}\right) d t \leq \frac{C\left(\boldsymbol{\rho}_{*}\right)}{2 T} N^{d-2}$. Therefore, if we set $H_{N}(f):=H\left(f d v_{\boldsymbol{\rho}_{*}}^{N} \mid v_{\boldsymbol{\rho}_{*}}^{N}\right)$, in order to prove the one-block estimate, it suffices to prove that for some $\rho_{*} \in A$

$$
\limsup _{\ell \rightarrow \infty} \limsup _{N \rightarrow \infty} \sup _{\substack{H_{N}(f) \leq C_{0} N^{d} \\ D_{N}(f) \leq C_{0} N^{d-2}}} \int \frac{1}{N^{d}} \sum_{x \in \mathbb{T}_{N}^{d}} \tau_{x} V^{\ell} f d v_{\boldsymbol{\rho}_{*}}^{N} \leq 0, \quad \forall C_{0}>0,
$$

where the supremum is taken among all densities $f \in L_{+}^{1}\left(v_{\boldsymbol{\rho}_{*}}^{N}\right)$.

In a second step, following the proof of the one-species case [19, Sect. 5.4] we cut off large densities. Since Lemma 4.1 requires only the $O\left(N^{d}\right)$-entropy assumption, it follows that

$$
\limsup _{N \rightarrow+\infty} \sup _{H_{N}(f) \leq C N^{d}} \frac{1}{N^{d}} \int|\eta|_{1} f d v_{\boldsymbol{\rho}_{*}}^{N}<+\infty, \text { for every } C>0 .
$$

Similarly to the one-species case, under the assumption that $g$ has sublinear growth at infinity in the sense of (27) (which always holds when $\boldsymbol{g}$ is bounded), inequality (32) allows us to cut off large densities, by restricting $V^{\ell}$ to the set of configurations $\eta$ which satisfy $\left|\eta^{\ell}(0)\right|_{1} \leq C_{1}$ 
for some constant $C_{1}>0$. This way the one-block estimate is reduced to proving that for all constants $C_{0}, C_{1}>0$

$$
\lim _{\ell \rightarrow+\infty} \limsup _{N \rightarrow+\infty} \sup _{D_{N}(f) \leq C_{0} N^{d-2}} \int \frac{1}{N^{d}} \sum_{x \in \mathbb{T}_{N}^{d}} \tau_{x} V^{\ell} \mathbb{1}_{\left\{\left|\eta^{\ell}(x)\right|_{1} \leq C_{1}\right\}} f d \nu_{\boldsymbol{\rho}_{*}}^{N} \leq 0 .
$$

In a third step, by adapting the steps 2 to 4 of [19, Sect. 5.4.1] to the two-species case, the one-block-estimate is further reduced to showing that for all constants $C_{1}>0$,

$$
\limsup _{\ell \rightarrow+\infty} \max _{\left.\boldsymbol{K}|| \boldsymbol{K}\right|_{1} \leq(2 \ell+1)^{d} C_{1}} \int V^{\ell} d \nu_{2 \ell+1, \boldsymbol{K}}=0,
$$

where the canonical measure $\nu_{2 \ell+1, K}$ is considered as a measure on $\mathrm{M}_{\infty}^{d}$ by identifying the cube $\Lambda_{\ell}^{d}:=\left\{x \in \mathbb{Z}^{d}|| x \mid \leq \ell\right\} \subseteq \mathbb{Z}^{d}$ with $\mathbb{T}_{2 \ell+1}^{d}$.

The final step in the proof of the one-block estimate consists in applying the equivalence of ensembles to prove (34). Since the measure $\nu_{2 \ell+1, K}$ is concentrated on configurations with $\boldsymbol{K}$ particles, the integral appearing in (34) is equal to

$$
\int V^{\ell} d \nu_{2 \ell+1, \boldsymbol{K}}=\int\left|\frac{1}{(2 \ell+1)^{d}} \sum_{|x| \leq \ell} \boldsymbol{g}(\boldsymbol{\xi}(x))-\overline{\boldsymbol{\Phi}}\left(\frac{\boldsymbol{K}}{(2 \ell+1)^{d}}\right)\right|_{1} d \nu_{2 \ell+1, \boldsymbol{K}} .
$$

As in the one-species case, by fixing a positive integer $k$ which will tend to infinity after taking the limit as $\ell \rightarrow+\infty$, and decomposing the cube $\Lambda_{\ell}^{d}$ in smaller cubes of side-length $2 k+1$, the one-block estimate is reduced to showing that

$$
\lim _{k \rightarrow \infty} \lim _{m \rightarrow \infty} S(m, k)=0,
$$

where $S(m, k)$ denotes the supremum

$$
S(m, k):=\sup _{\substack{\ell \geq m \\|\boldsymbol{K}|_{1} \leq(2 \ell+1)^{d} C_{1}}} \int\left|\frac{1}{(2 k+1)^{d}} \sum_{|x| \leq k} \boldsymbol{g}(\xi(x))-\overline{\boldsymbol{\Phi}}\left(\frac{\boldsymbol{K}}{(2 \ell+1)^{d}}\right)\right|_{1} d \nu_{2 \ell+1, \boldsymbol{K}} .
$$

This is the part of the proof where we need the boundedness and the regularity of the tails (18) of the jump rate $g$ as well as the continuity of the partition function $Z$ on $\mathcal{D}_{Z} \cap \partial \mathcal{D}_{Z}$. For each fixed $(m, k) \in \mathbb{N} \times \mathbb{N}$, we pick a sequence $\left\{\left(\ell_{n}^{m, k}, \boldsymbol{K}_{n}^{m, k}\right)\right\}_{n \in \mathbb{N}}$ such that $\ell_{n}^{m, k} \geq m$ and $\left|\boldsymbol{K}_{n}^{m, k}\right|_{1} \leq\left(2 \ell_{n}^{m, k}+1\right)^{d} C_{1}$ for all $n \in \mathbb{N}$ that achieves the supremum, i.e., such that

$$
S(m, k)=\lim _{n \rightarrow \infty} \int\left|\frac{1}{(2 k+1)^{d}} \sum_{|x| \leq k} \boldsymbol{g}(\boldsymbol{\xi}(x))-\overline{\boldsymbol{\Phi}}\left(\frac{\boldsymbol{K}_{n}^{m, k}}{\left(2 \ell_{n}^{m, k}+1\right)^{d}}\right)\right|_{1} d v_{2 \ell_{n}^{m, k}+1, \boldsymbol{K}_{n}^{m, k}}
$$

Since the sequence $\left\{\boldsymbol{r}_{n}^{m, k}\right\}_{n \in \mathbb{N}}$ defined by

$$
\boldsymbol{r}_{n}^{m, k}:=\frac{\boldsymbol{K}_{n}^{m, k}}{\left(2 \ell_{n}^{m, k}+1\right)^{d}}, \quad n \in \mathbb{N},
$$

is contained in the compact triangular region $B_{|\cdot|_{1}}\left(0, C_{1}\right):=\left\{\left.\boldsymbol{r} \in \mathbb{R}_{+}^{2}|| \boldsymbol{r}\right|_{1} \leq C_{1}\right\}$, for each fixed $(m, k) \in \mathbb{N} \times \mathbb{N}$, we can pick a sequence $\left\{n_{j}\right\}_{j \in \mathbb{N}}:=\left\{n_{j}^{m, k}\right\}$ such that $\boldsymbol{r}_{n_{j}}^{m, k}$ converges to some $\boldsymbol{r}^{m, k} \in B_{|\cdot|_{1}}\left(0, C_{1}\right)$ as $j \rightarrow \infty$. Since we assume that $\boldsymbol{g}$ is bounded, it follows by the equivalence of ensembles that

$$
S(m, k)=\int\left|\frac{1}{(2 k+1)^{d}} \sum_{|x| \leq k} \boldsymbol{g}(\boldsymbol{\xi}(x))-\overline{\boldsymbol{\Phi}}\left(\boldsymbol{r}^{m, k}\right)\right|_{1} d \nu_{\boldsymbol{R}_{c}\left(\boldsymbol{r}^{m, k}\right)}^{\infty} .
$$


Furthermore, since $\left|\boldsymbol{R}_{c}(\boldsymbol{\rho})\right|_{1} \leq|\boldsymbol{\rho}|_{1}$, for each fixed $k \in \mathbb{N}$ the sequence $\left\{\boldsymbol{\rho}^{m, k}:=\right.$ $\left.\boldsymbol{R}_{c}\left(\boldsymbol{r}^{m, k}\right)\right\}_{m \in \mathbb{N}}$, is also contained in $B_{|\cdot|_{1}}\left(0, C_{1}\right)$ and thus we can choose a sequence $\left\{m_{j}\right\}_{j \in \mathbb{N}}=\left\{m_{j}^{(k)}\right\}$ such that $\left\{\boldsymbol{\rho}^{m_{j}, k}\right\}_{m \in \mathbb{N}}$ converges to some $\boldsymbol{\rho}^{k} \in B_{|\cdot|_{1}}\left(0, C_{1}\right) \cap \boldsymbol{R}\left(\mathcal{D}_{\boldsymbol{R}}\right)$. By the continuity assumption on $Z$, the grand canonical ensemble is weakly continuous. By this fact, the continuity of $\boldsymbol{R}_{c}$ and the identity $\overline{\boldsymbol{\Phi}}=\boldsymbol{\Phi} \circ \boldsymbol{R}_{c}$,

$$
\lim _{m \rightarrow \infty} S(m, k)=\int\left|\frac{1}{(2 k+1)^{d}} \sum_{|x| \leq k} \boldsymbol{g}(\xi(x))-\boldsymbol{\Phi}\left(\rho^{k}\right)\right|_{1} d \nu_{\rho^{k}}^{\infty} .
$$

Therefore

$$
\limsup _{k \rightarrow+\infty} \lim _{m \rightarrow+\infty} S(m, k) \leq \limsup _{k \rightarrow \infty} \sup _{\boldsymbol{\rho} \in \boldsymbol{R}\left(\mathcal{D}_{\boldsymbol{R}}\right)} \int\left|\frac{1}{(2 k+1)^{d}} \sum_{|x| \leq k} \boldsymbol{g}(\boldsymbol{\eta}(x))-\boldsymbol{\Phi}(\boldsymbol{\rho})\right|_{1} d \nu_{\boldsymbol{\rho}}^{\infty} .
$$

The random variables $\boldsymbol{g}(\boldsymbol{\eta}(x)), x \in \mathbb{Z}^{d}$, are uniformly bounded by $\|\boldsymbol{g}\|_{\infty}$ and i.i.d. with respect to $v_{\rho}^{\infty}$ for all $\rho \in \boldsymbol{R}\left(\mathcal{D}_{\boldsymbol{R}}\right)$ and thus they satisfy the $L^{2}$-weak law of large numbers uniformly over all parameters $\rho \in \boldsymbol{R}\left(\mathcal{D}_{\boldsymbol{R}}\right)$, which shows that the term in the right hand side above is equal to zero. This completes the proof of the one-block estimate and hence the proof of Theorem 3.1.

\subsection{Proof of Theorem 3.2}

Let $A$ be the interior of the set of all strictly positive sub-critical densities, i.e.,

$$
A:=\boldsymbol{R}\left(\mathcal{D}_{\boldsymbol{R}}^{o}\right) \cap(0, \infty)^{2},
$$

and let $\rho:\left[0, T_{\max }\right) \times \mathbb{T}^{d} \rightarrow A$ be the maximal classical solution established in [1] of the initial value problem (1) with $\rho(0, \cdot):=\rho_{0} \in C^{2+\theta}\left(\mathbb{T}^{d} ; A\right)$. We fix $\boldsymbol{a} \in A$ and denote by $\psi_{t}^{N}$ the Radon-Nikodym derivative of $v_{\boldsymbol{\rho}_{t}(\cdot)}^{N}$ with respect to $v_{\boldsymbol{a}}^{N}$,

$$
\psi_{t}^{N}:=\frac{d v_{\boldsymbol{\rho}_{t}(\cdot)}^{N}}{d v_{\boldsymbol{a}}^{N}}
$$

Let $H_{N}(t):=\mathcal{H}\left(\mu_{t}^{N} \mid v_{\rho_{t}(\cdot)}^{N}\right)$ be the relative entropy of $\mu_{t}^{N}$ with respect to $v_{\rho_{t}(\cdot)}^{N}$. We have the following upper bound on the entropy production, proved in [19, Lemma 6.1.4],

$$
\partial_{t} H_{N}(t) \leq \int \frac{1}{\psi_{t}^{N}}\left\{N^{2} L_{N}^{*} \psi_{t}^{N}-\partial_{t} \psi_{t}^{N}\right\} d \mu_{t}^{N}
$$

for every $t \in\left[0, T_{\max }\right)$, where $L_{N}^{*}$ is the adjoint of $L_{N}$ in $L^{2}\left(v_{\boldsymbol{a}}^{N}\right)$. Denoting by

$$
H(t):=\limsup _{N \rightarrow \infty} \frac{1}{N^{d}} H_{N}(t), \quad t \in\left[0, T_{\max }\right),
$$

the limiting entropy density, the main step in the application of the relative entropy method is to use this upper bound on $\partial_{t} H_{N}(t)$ to get an inequality of the form

$$
H(t) \leq H(0)+\frac{1}{\gamma} \int_{0}^{t} H(s) d s
$$

for some constant $\gamma>0$. Since $H(0)=0$ by assumption, this implies by Gronwall's inequality that $H(t)=0$ for all $t \in\left[0, T_{\max }\right)$ as required. Of course, in order for Gronwall's inequality to be applicable, $H$ must belong at least in $L_{\text {loc }}^{1}\left(\left[0, T_{\max }\right)\right]$. This is the context of the next two lemmas. The first is Remark 6.1.2 in [19] for single-species ZRPs. 
Lemma 4.2 If $\left\{\mu_{0}^{N}\right\}$ is an entropy local equilibrium of profile $\rho \in C\left(\mathbb{T}^{d} ; \boldsymbol{R}\left(\mathcal{D}_{\boldsymbol{R}}^{o}\right)\right)$, then $\left\{\mu_{0}^{N}\right\}$ satisfies the $O\left(N^{d}\right)$-entropy assumption (28).

Proof Indeed, for fixed $\boldsymbol{a} \in A:=\boldsymbol{R}\left(\mathcal{D}_{\boldsymbol{R}}^{o}\right) \cap(0, \infty)^{2}$, by the relative entropy inequality [19, Sect. A.1.8]

$$
\mathcal{H}\left(\mu_{0}^{N} \mid v_{\boldsymbol{a}}^{N}\right) \leq\left(1+\frac{1}{\gamma}\right) \mathcal{H}\left(\mu_{0}^{N} \mid v_{\boldsymbol{\rho}(\cdot)}^{N}\right)+\frac{1}{\gamma} \log \int e^{\gamma \log \frac{d v_{\rho(\cdot)}^{N}}{d v_{\boldsymbol{a}}^{N}}} d v_{\boldsymbol{\rho}(\cdot)}^{N} .
$$

Since $v_{\boldsymbol{\rho}(\cdot)}^{N}, v_{\boldsymbol{a}}^{N}$ are product measures, the Radon-Nikodym derivative $\frac{d v_{\rho(\cdot)}^{N}}{d v_{\boldsymbol{a}}^{N}}$ can be computed explicitly. With the notation $\boldsymbol{\Phi}_{\boldsymbol{a}}:=\left(\frac{\Phi_{1}}{\Phi_{1}(\boldsymbol{a})}, \frac{\Phi_{2}}{\Phi_{2}(\boldsymbol{a})}\right), Z_{\boldsymbol{a}}:=\frac{Z \circ \boldsymbol{\Phi}}{Z(\boldsymbol{\Phi}(\boldsymbol{a}))}$

$$
\int\left(\frac{d \nu_{\boldsymbol{\rho}(\cdot)}^{N}}{d \nu_{\boldsymbol{a}}^{N}}\right)^{\gamma} d \nu_{\boldsymbol{\rho}(\cdot)}^{N}=\prod_{x \in \mathbb{T}_{N}^{d}} \frac{1}{Z_{\boldsymbol{a}}(\boldsymbol{\rho}(x / N))^{\gamma}} \int e^{\left\langle\boldsymbol{k}, \gamma \log \boldsymbol{\Phi}_{\boldsymbol{a}}(\boldsymbol{\rho}(x / N))\right\rangle} d \nu_{\boldsymbol{\rho}(x / N)}^{1}(\boldsymbol{k}) .
$$

Since $Z \geq 1$, we have that $\frac{1}{Z_{\boldsymbol{a}}(\boldsymbol{\rho})}=\frac{Z(\boldsymbol{\Phi}(\boldsymbol{a}))}{Z(\boldsymbol{\Phi}(\boldsymbol{\rho}))} \leq Z(\boldsymbol{\Phi}(\boldsymbol{a}))$ and therefore

$$
\begin{aligned}
\frac{1}{\gamma N^{d}} \log \int\left(\frac{d \nu_{\boldsymbol{\rho}(\cdot)}^{N}}{d \nu_{\boldsymbol{a}}^{N}}\right)^{\gamma} d \nu_{\boldsymbol{\rho}(\cdot)}^{N} & \leq Z(\boldsymbol{\Phi}(\boldsymbol{a}))+\frac{1}{\gamma N^{d}} \sum_{x \in \mathbb{T}_{N}^{d}} \Lambda_{\rho(x / N)}\left(\gamma \log \boldsymbol{\Phi}_{\boldsymbol{a}}(\rho(x / N))\right) \\
& \leq Z(\boldsymbol{\Phi}(\boldsymbol{a}))+\frac{1}{\gamma N^{d}} \sum_{x \in \mathbb{T}_{N}^{d}} \log Z\left(\boldsymbol{F}_{\boldsymbol{a}}(x / N, \gamma)\right)
\end{aligned}
$$

where here $\boldsymbol{F}_{\boldsymbol{a}}: \mathbb{T}^{d} \times[0,1] \rightarrow(0, \infty)^{2}$ is the function given by $\boldsymbol{F}_{\boldsymbol{a}}(u, \gamma)=\frac{\boldsymbol{\Phi}(\boldsymbol{\rho}(u))^{1+\gamma}}{\boldsymbol{\Phi}(\boldsymbol{a})^{\gamma}}$ and for $\boldsymbol{a} \in \mathbb{R}_{+}^{2}, \boldsymbol{b} \in(0, \infty)^{2}, \gamma>0$, we have set $\boldsymbol{a}^{\gamma}:=\left(a_{1}^{\gamma}, a_{2}^{\gamma}\right)$ and $\frac{\boldsymbol{a}}{\boldsymbol{b}}:=\left(\frac{a_{1}}{b_{1}}, \frac{a_{2}}{b_{2}}\right)$. Since $\rho\left(\mathbb{T}^{d}\right) \subseteq \boldsymbol{R}\left(\mathcal{D}_{\boldsymbol{R}}^{o}\right)$ by assumption, it follows that $\boldsymbol{\Phi}\left(\rho\left(\mathbb{T}^{d}\right)\right) \subseteq \mathcal{D}_{Z}^{o}$. Since $\boldsymbol{F}_{\boldsymbol{a}}$ is uniformly continuous on $\mathbb{T}^{d} \times[0,1]$ and satisfies $\lim _{\gamma \rightarrow 0} \boldsymbol{F}_{\boldsymbol{a}}(u, \gamma)=\boldsymbol{\Phi}(\boldsymbol{\rho}(u))$ for all $u \in \mathbb{T}^{d}$, it follows that its image is contained in $\mathcal{D}_{Z}^{o}$, i.e., $\left\{\boldsymbol{F}_{\boldsymbol{a}}(u, \gamma) \mid u \in \mathbb{T}^{d}\right\} \subseteq \mathcal{D}_{Z}^{o}$ for sufficiently small $\gamma>0$. Then the function $u \mapsto Z\left(\boldsymbol{F}_{\boldsymbol{a}}(u, \gamma)\right)$ is well defined and continuous on the torus $\mathbb{T}^{d}$, so that its Riemannian sums converge. By (39), (40) and the fact that $\mu_{0}^{N}$ is an entropy local equilibrium, this yields that

$$
C(\boldsymbol{a}) \leq Z(\boldsymbol{\Phi}(\boldsymbol{a}))+\frac{1}{\gamma} \int_{\mathbb{T}^{d}} \log Z\left(\boldsymbol{F}_{\boldsymbol{a}}(u, \gamma)\right) d u<+\infty
$$

for small $\gamma>0$, and the proof of Lemma 4.2 is complete.

Lemma 4.3 Let $\rho:[0, T] \times \mathbb{T}^{d} \rightarrow \boldsymbol{R}\left(\mathcal{D}_{\boldsymbol{R}}^{o}\right) \cap(0, \infty)^{2}$ be a continuous function and let $\left\{\mu_{0}^{N}\right\}$ be an entropy local equilibrium with respect to $\rho_{0}:=\rho(0, \cdot)$. Then the upper entropy $\bar{H}:[0, T] \rightarrow[0,+\infty]$ defined by

$$
\bar{H}(t):=\sup _{N \in \mathbb{N}} \frac{1}{N^{d}} \mathcal{H}\left(\mu_{t}^{N} \mid v_{\rho_{t}(\cdot)}^{N}\right)
$$

belongs to $L^{\infty}([0, T])$.

Proof By the relative entropy inequality and [19, Proposition A.1.9.1], according to which the function $t \mapsto \mathcal{H}\left(\mu_{t}^{N} \mid \nu_{\boldsymbol{a}}^{N}\right)$ is non-increasing,

$$
H_{N}(t) \leq\left(1+\frac{1}{\gamma}\right) \mathcal{H}\left(\mu_{0}^{N} \mid v_{\boldsymbol{a}}^{N}\right)+\frac{1}{\gamma} \log \int\left(\frac{d v_{\boldsymbol{a}}^{N}}{d \nu_{\boldsymbol{\rho}_{t}(\cdot)}^{N}}\right)^{\gamma} d v_{\boldsymbol{a}}^{N}
$$


for all $t \geq 0$ and all $\gamma>0$. Since the proper domain of $Z_{a}$ has interior $\mathcal{D}_{Z_{a}}^{o}=\boldsymbol{R}\left(\mathcal{D}_{\boldsymbol{R}}^{o}\right)$ and since $\rho\left([0, T] \times \mathbb{T}^{d}\right) \subseteq \boldsymbol{R}\left(\mathcal{D}_{\boldsymbol{R}}^{o}\right) \cap(0, \infty)^{2}$, the function $Z_{\boldsymbol{a}} \circ \rho$ is a bounded continuous function on the torus, and therefore, by a computation similar to the one in the proof of Lemma 4.2, we obtain

$$
\begin{aligned}
& \frac{1}{\gamma N^{d}} \log \int\left(\frac{d v_{\boldsymbol{a}}^{N}}{d v_{\boldsymbol{\rho}_{t}(\cdot)}^{N}}\right)^{\gamma} d v_{\boldsymbol{a}}^{N} \\
& =\left\|Z_{\boldsymbol{a}} \circ \boldsymbol{\rho}\right\|_{L^{\infty}\left([0, T] \times \mathbb{T}^{d}\right)}+\frac{1}{\gamma N^{d}} \sum_{x \in \mathbb{T}_{N}^{d}} \Lambda_{\boldsymbol{a}}\left(\gamma \log \frac{1}{\boldsymbol{\Phi}_{\boldsymbol{a}}\left(\boldsymbol{\rho}_{t}(x / N)\right)}\right),
\end{aligned}
$$

where $\left\|Z_{\boldsymbol{a}} \circ \rho\right\|_{L^{\infty}\left([0, T] \times \mathbb{T}^{d}\right)}<+\infty$. For the second term, we have for every $u \in \mathbb{T}^{d}$ that

$$
\Lambda_{\boldsymbol{a}}\left(\gamma \log \frac{1}{\boldsymbol{\Phi}_{\boldsymbol{a}}\left(\boldsymbol{\rho}_{t}(u)\right)}\right)=\log \left\{\frac{1}{Z(\boldsymbol{\Phi}(\boldsymbol{a}))} Z\left(\frac{\boldsymbol{\Phi}(\boldsymbol{a})^{1+\gamma}}{\boldsymbol{\Phi}\left(\boldsymbol{\rho}_{t}(u)\right)^{\gamma}}\right)\right\} .
$$

Since $\boldsymbol{\Phi}(\rho)\left(\mathbb{T}^{d}\right) \subseteq(0, \infty)^{2}$ and $\boldsymbol{\Phi}(\rho)$ is continuous, there exists $\varphi_{0} \in \mathcal{D}_{Z}$ such that $\varphi_{0}<$ $\boldsymbol{\Phi}(\rho(t, u))$ for all $(t, u) \in[0, T] \times \mathbb{T}^{d}$. Then since $Z$ is increasing,

$$
Z\left(\frac{\boldsymbol{\Phi}(\boldsymbol{a})^{1+\gamma}}{\boldsymbol{\Phi}\left(\rho_{t}(u)\right)^{\gamma}}\right) \leq Z\left(\frac{\boldsymbol{\Phi}(\boldsymbol{a})^{1+\gamma}}{\boldsymbol{\varphi}_{0}^{\gamma}}\right)
$$

and since $\boldsymbol{\Phi}(\boldsymbol{a}) \in \mathcal{D}_{Z}^{o}$ and $\boldsymbol{\Phi}(\boldsymbol{a})^{1+\gamma} / \boldsymbol{\varphi}_{0}^{\gamma} \rightarrow \boldsymbol{\Phi}(\boldsymbol{a})$ as $\gamma \rightarrow 0$, we can choose $\gamma_{0}>0$ sufficiently small so that $\boldsymbol{\Phi}(\boldsymbol{a})^{1+\gamma} / \boldsymbol{\varphi}_{0}^{\gamma} \in \mathcal{D}_{Z}^{o}$ and $Z\left(\boldsymbol{\Phi}(\boldsymbol{a})^{1+\gamma} / \boldsymbol{\varphi}_{0}^{\gamma}\right) \leq Z(\boldsymbol{\Phi}(\boldsymbol{a}))+1$ for all $\gamma<\gamma_{0}$. Consequently, since by Lemma $4.2\left\{\mu_{0}^{N}\right\}$ satisfies the $O\left(N^{d}\right)$-entropy assumption, by (41) for some constant $C \geq 0$ for all $\gamma<\gamma_{0}$

$$
\|\bar{H}\|_{L^{\infty}([0, T])} \leq\left(1+\frac{1}{\gamma}\right) C+\left\|Z_{\boldsymbol{a}}(\boldsymbol{\rho})\right\|_{L^{\infty}\left([0, T] \times \mathbb{T}^{d}\right)}+\frac{1}{\gamma} \log \frac{Z(\boldsymbol{\Phi}(\boldsymbol{a}))+1}{Z(\boldsymbol{\Phi}(\boldsymbol{a}))}<+\infty,
$$

establishing the claim of Lemma 4.3.

The bound (36) on the entropy production can be estimated explicitly. Since $v_{\boldsymbol{\rho}_{t}(\cdot)}^{N}, v_{\boldsymbol{a}}^{N}$ are product measures, $\psi_{t}$ can be computed explicitly. Then by differentiating, using the chain rule, the fact that $\rho$ is a solution of the hydrodynamic equation, the relations $\frac{\varphi_{i} \partial_{i} Z(\varphi)}{Z(\varphi)}=R_{i}(\varphi)$, $i=1,2$ and the relation (13) we obtain

$$
\frac{\partial_{t} \psi_{t}^{N}}{\psi_{t}^{N}}=\sum_{x \in \mathbb{T}_{N}^{d}}\left\langle\frac{\Delta \boldsymbol{\Phi}\left(\rho_{t}(x / N)\right)}{\boldsymbol{\Phi}\left(\rho_{t}(x / N)\right)}, D \boldsymbol{\Phi}\left(\rho_{t}(x / N)\right)\left[\eta(x)-\rho_{t}(x / N)\right]\right\rangle .
$$

We note here that as in the asymmetric case treated in [16], at this point of the application of the relative entropy method for two-species ZRPs one has to use the macroscopic analogue (13) of the compatibility relations (6).

For the other term, by computations of the action of the generator on $\psi_{t}^{N}$ similar to the ones for the single-species case in [19],

$$
\frac{L_{N}^{*} \psi_{t}^{N}}{\psi_{t}^{N}}=\sum_{i=1,2} \sum_{x, y \in \mathbb{T}_{N}^{d}}\left[\frac{\Phi_{i}\left(\rho_{t}(y / N)\right)}{\Phi_{i}\left(\rho_{t}(x / N)\right)}-1\right]\left[g_{i}(\eta(x))-\Phi_{i}\left(\rho_{t}(x / N)\right)\right] p(y-x) .
$$


Since $\boldsymbol{\Phi}\left(\boldsymbol{\rho}_{t}\right)$ is $C^{2+\theta}$ for some $\theta>0$ and the n.n. transition probability has mean zero, the Taylor expansion for $C^{2+\theta}$ functions yields (with the renormalisation $p\left(\mathbb{Z}^{d}\right)=2 d$ ) that

$$
\frac{N^{2} L_{N}^{*} \psi_{t}^{N}}{\psi_{t}^{N}}=\sum_{x \in \mathbb{T}_{N}^{d}}\left\langle\frac{\Delta\left[\boldsymbol{\Phi}\left(\boldsymbol{\rho}_{t}\right)\right]}{\boldsymbol{\Phi}\left(\boldsymbol{\rho}_{t}\right)}\left(\frac{x}{N}\right), \boldsymbol{g}(\boldsymbol{\eta}(x))-\boldsymbol{\Phi}\left(\boldsymbol{\rho}_{t}(x / N)\right)\right\rangle+r_{N}(t) .
$$

Here, for any $T \in\left[0, T_{\max }\right)$, the remainder $r_{N}(t)$ satisfies the bound

$$
\left|r_{N}(t)\right| \leq \frac{C_{T} \boldsymbol{g}^{*}}{N^{\theta}}|\boldsymbol{\eta}|_{1}+\frac{C_{T} M_{T}}{m_{T}} N^{d-\theta}
$$

for all $t \in[0, T]$, where $\boldsymbol{g}^{*}$ is the constant in (4), $C_{T}=C(d, p, \boldsymbol{\Phi}(\boldsymbol{\rho}), T) \geq 0$ is the constant

$$
C_{T}=\sqrt{d} \sup _{0 \leq t \leq T}\left\|D^{2}\left[\Phi_{1}\left(\rho_{t}\right)\right]\right\|_{C^{\theta}} \vee\left\|D^{2}\left[\Phi_{2}\left(\rho_{t}\right)\right]\right\|_{C^{\theta}} \sum_{y \in \mathbb{Z}^{d}}\|y\|^{2+\theta} p(y)
$$

with $\|\cdot\|_{C^{\theta}}$ denoting the $\theta$-Hölder seminorm and

$m_{T}:=\inf _{(t, u) \in[0, T] \times \mathbb{T}^{d}} \min _{i=1,2} \Phi_{i}\left(\rho_{t}(u)\right)>0, \quad M_{T}:=\sup _{(t, u) \in[0, T] \times \mathbb{T}^{d}}|\boldsymbol{\Phi}(\rho(t, u))|_{1}<+\infty$.

By this bound on the remainder and the conservation of the number of particles it follows that for all $t \in[0, T] \subseteq\left[0, T_{\max }\right), T>0$,

$$
\frac{1}{N^{d}} \int_{0}^{t} \int r_{N}(t) d \mu_{t}^{N} d t \leq \frac{C_{T} \boldsymbol{g}^{*} t}{N^{d+\theta}} \int|\boldsymbol{\eta}|_{1} d \mu_{0}^{N}+\frac{C_{T} M_{T} t}{m_{T}} \frac{1}{N^{\theta}},
$$

which according to Lemma 4.1 shows that

$$
\int_{0}^{t} \int r_{N}(s) d \mu_{s}^{N} d s \leq o\left(N^{d}\right)
$$

Since the function $\frac{\Delta\left[\Phi\left(\rho_{t}\right)\right]}{\Phi\left(\rho_{t}\right)}$ is in $C_{\mathrm{loc}}\left(\left[0, T_{\max }\right) \times \mathbb{T}^{d}\right)$, a change of variables shows that

$$
\int_{0}^{t} \int \sum_{x \in \mathbb{T}_{N}^{d}}\left\langle\frac{\Delta\left[\boldsymbol{\Phi}\left(\boldsymbol{\rho}_{s}\right)\right]}{\boldsymbol{\Phi}\left(\boldsymbol{\rho}_{s}\right)}\left(\frac{x}{N}\right), \boldsymbol{\eta}(x)-\eta^{\ell}(x)\right\rangle d \mu_{s}^{N} d s=o\left(N^{d}\right)
$$

for all $t \in\left[0, T_{\max }\right)$. Integrating (36) in time, using the explicit expressions (43), (45), taking into account (46) and the fact that $\left\{\mu_{0}^{N}\right\}$ is an entropy local equilibrium (i.e., (20) holds) and using (47) and the one-block estimate, one obtains that for all $t \in\left(0, T_{\max }\right)$

$$
H_{N}(t) \leq \int_{0}^{t} \int \sum_{x \in \mathbb{T}_{N}^{d}}\left\langle\frac{\Delta\left[\boldsymbol{\Phi}\left(\rho_{s}\right)\right]}{\boldsymbol{\Phi}\left(\rho_{s}\right)}\left(\frac{x}{N}\right), \boldsymbol{\Psi}\left(\rho_{s}(x / N), \eta^{\ell}(x)\right)\right) d \mu_{s}^{N} d s+o_{\ell}\left(N^{d}\right),
$$

where $\boldsymbol{\Psi}: \boldsymbol{R}\left(\mathcal{D}_{\boldsymbol{R}}^{o}\right) \times \mathbb{R}_{+}^{2} \rightarrow \mathbb{R}^{2}$ is the quasi-potential

$$
\boldsymbol{\Psi}(\rho, \lambda)=\overline{\boldsymbol{\Phi}}(\lambda)-\boldsymbol{\Phi}(\rho)-D \boldsymbol{\Phi}(\rho)(\lambda-\rho)
$$

and the term $o_{\ell}\left(N^{d}\right)$ satisfies $o_{\ell}\left(N^{d}\right) / N^{d} \rightarrow 0$ as $N$ and then $\ell$ tend to infinity. In the definition of the quasi-potential the second variable $\lambda$ is in $\mathbb{R}_{+}^{2}$ since it is to be substituted by the large microscopic averages $\eta^{\ell}(x), x \in \mathbb{T}_{N}^{d}$. Thus the extension $\overline{\boldsymbol{\Phi}}$ of $\boldsymbol{\Phi}$ must be used in the quasi-potential. To simplify the notation, we set

$$
G_{t}(u, \lambda):=\left\langle\frac{\Delta\left[\boldsymbol{\Phi}\left(\rho_{t}\right)\right]}{\boldsymbol{\Phi}\left(\rho_{t}\right)}(u), \boldsymbol{\Psi}\left(\rho_{t}(u), \lambda\right)\right\rangle .
$$


By the relative entropy inequality, we have for all $\gamma>0$ and all $0 \leq s<T_{\max }$ that

$$
\int \sum_{x \in \mathbb{T}_{N}^{d}} G_{s}\left(x / N, \eta^{\ell}(x)\right) d \mu_{s}^{N} \leq \frac{1}{\gamma} H_{N}(s)+\frac{1}{\gamma} \log \int e^{\gamma \sum_{x \in \mathbb{T}_{N}^{d}} G_{s}\left(\frac{x}{N}, \eta^{\ell}(x)\right)} d \nu_{\boldsymbol{\rho}_{s}(\cdot)}^{N} .
$$

By combining this inequality with the bound (48), dividing by $N^{d}$ and taking the lim sup as $N \rightarrow \infty$ and then $\ell \rightarrow \infty$, we get

$$
H(t) \leq \frac{1}{\gamma} \int_{0}^{t} H(s) d s+\limsup _{\ell, N \rightarrow+\infty} \frac{1}{\gamma N^{d}} \int_{0}^{t} \log \int e^{\gamma \sum_{x \in \mathbb{T}_{N}^{d}} G_{s}\left(\frac{x}{N}, \eta^{\ell}(x)\right)} d v_{\rho_{s}(\cdot)}^{N} d s,
$$

where in order to obtain the term $\int_{0}^{t} H(s) d s$ we used Lemma 4.3 to pass the limit inside the integral and $\lim \sup _{\ell, N \rightarrow+\infty}$ denotes the lim sup as $N \rightarrow+\infty$ and then $\ell \rightarrow+\infty$.

To complete the proof of Theorem 3.2, it remains to show that for each $t \in\left[0, T_{\max }\right)$ we can choose $\gamma>0$ small enough so that the rightmost term in (51) vanishes. We begin by noting that the function $G:\left[0, T_{\max }\right) \times \mathbb{T}^{d} \times \mathbb{R}_{+}^{2} \rightarrow \mathbb{R}$ defined in (50) satisfies

$$
\left|G_{t}(u, \lambda)\right| \leq\left|\frac{\Delta\left[\boldsymbol{\Phi}\left(\rho_{t}\right)\right]}{\boldsymbol{\Phi}\left(\rho_{t}\right)}(u)\right|_{\infty}\left\{g^{*}\left(|\lambda|_{1}+\left|\left(\rho_{t}(u)\right)\right|_{1}\right)+\left|D \boldsymbol{\Phi}\left(\rho_{t}(u)\right)\right|_{\infty}\left(|\lambda|_{1}+\left|\rho_{t}(u)\right|_{1}\right)\right\}
$$

for all $(t, u, \lambda) \in\left[0, T_{\max }\right) \times \mathbb{T}^{d} \times \mathbb{R}_{+}^{2}$, which for any $t \in\left[0, T_{\max }\right)$ yields the inequality

$$
\sup _{(s, u) \in[0, t] \times \mathbb{T}^{d}}\left|G_{s}(u, \lambda)\right| \leq C_{t} \cdot\left(1+|\lambda|_{1}\right) \text { for all } \lambda \in \mathbb{R}_{+}^{2}
$$

for some constant $C_{t}<+\infty$. Since for any $t \in\left[0, T_{\max }\right)$ we have $\rho\left([0, t] \times \mathbb{T}^{d}\right) \subseteq \boldsymbol{R}\left(\mathcal{D}_{\boldsymbol{R}}^{o}\right)$ the set $\boldsymbol{\Phi}(\rho)\left([0, T] \times \mathbb{T}^{d}\right)$ is bounded away from the critical densities $\boldsymbol{\varphi}_{c} \in \partial \mathcal{D}_{Z}$, and thus there exists $\varepsilon>0$ such that

$$
\sup _{(t, u) \in[0, T] \times \mathbb{T}^{d}} \Lambda_{\rho(t, u)}(\lambda)<+\infty, \quad \forall \lambda \in D(0, \varepsilon),
$$

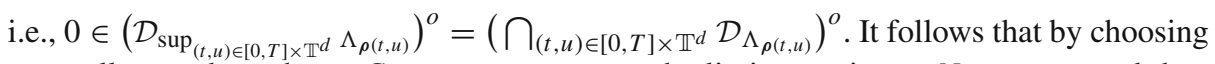
$\gamma_{t}$ small enough so that $\gamma_{t} C_{t}<\varepsilon$, we can pass the limit superior as $N \rightarrow+\infty$ and then $\ell \rightarrow+\infty$ inside the time integral in the rightmost term in (51). Thus in order to complete the proof it remains to show that for each $t \in\left[0, T_{\max }\right)$ we can choose $\gamma_{t}>0$ small enough so that

$$
\limsup _{\ell, N \rightarrow+\infty} \frac{1}{\gamma_{t} N^{d}} \log \int e^{\gamma_{t} \sum_{x \in \mathbb{T}_{N}^{d}} G_{t}\left(\frac{x}{N}, \eta^{\ell}(x)\right)} d v_{\boldsymbol{\rho}_{t}(\cdot)}^{N} \leq 0 .
$$

The proof of (53) relies on a corollary of the Laplace-Varadhan lemma [4, Sect. 4.3] for the large deviations principle satisfied by the independent family of the occupation variables $\{\boldsymbol{\eta}(x)\}_{x \in \mathbb{Z}^{d}}$ with respect to the invariant measure $v_{\rho}^{\infty}$ on the infinite lattice $\mathbb{Z}^{d}$ for some $\rho \in A$. Since the one-site marginal $v_{\rho}^{1}$ has some exponential moments for $\rho \in A$, by Cramér's theorem, the large deviations functional of the family $\{\boldsymbol{\eta}(x)\}_{x \in \mathbb{Z}^{d}}$ is given by the Legendre transform $\Lambda_{\rho}^{*}$ of the logarithmic moment-generating functional $\Lambda_{\rho}$. Note that (15) implies that modulo an affine function depending on $\rho$, the rate functional $\Lambda_{\rho}^{*}$ coincides with the thermodynamic entropy $S$, that is

$$
\Lambda_{\rho}^{*}(\lambda)=S(\lambda)-\langle\lambda, \log \boldsymbol{\Phi}(\rho)\rangle+\log Z(\boldsymbol{\Phi}(\rho)) .
$$


Lemma 4.4 Let $\rho: \mathbb{T}^{d} \rightarrow \boldsymbol{R}\left(\mathcal{D}_{\boldsymbol{R}}^{o}\right)$ be a continuous profile and let $G: \mathbb{T}^{d} \times \mathbb{R}_{+} \rightarrow \mathbb{R}$ be a continuous function such that

$$
\sup _{u \in \mathbb{T}^{d}}|G(u, \lambda)| \leq C\left(1+|\lambda|_{1}\right) \text { for all } \lambda \in \mathbb{R}_{+}^{2}
$$

for some constant $C>0$ such that $(2 C, 2 C) \in\left(\bigcap_{u \in \mathbb{T}^{d}} \mathcal{D}_{\Lambda_{\rho(u)}}\right)^{o}$. Then

$\limsup _{\ell \rightarrow \infty} \limsup _{N \rightarrow \infty} \frac{1}{N^{d}} \log \int e^{\sum_{x \in \mathbb{T}_{N}^{d}} G\left(\frac{x}{N}, \eta^{\ell}(x)\right)} d v_{\rho(\cdot)}^{N} \leq \int_{\mathbb{T}^{d}} \sup _{\lambda \in \mathbb{R}_{+}^{2}}\left\{G(u, \lambda)-\frac{1}{2} \Lambda_{\rho(u)}^{*}(\lambda)\right\} d u$.

We omit the proof of this Lemma as it is a simple adaptation of the corresponding result in the one-species case, [19, Lemma 6.1.10]. By the bound (52) the function $G:\left[0, T_{\max }\right) \times$ $\mathbb{T}^{d} \times \mathbb{R}_{+}^{2} \rightarrow \mathbb{R}$ defined in (50) satisfies

$$
\sup _{u \in \mathbb{T}^{d}}\left|G_{t}(u, \lambda)\right| \leq C_{t}\left(1+|\lambda|_{1}\right)
$$

for each fixed $t \in\left[0, T_{\max }\right)$. Therefore, if we choose $\gamma_{t}>0$ small enough so that $2 \gamma_{t} C_{t}\left(\boldsymbol{e}_{1}+\right.$ $\left.\boldsymbol{e}_{2}\right) \in\left(\bigcap_{u \in \mathbb{T}^{d}} \Lambda_{\rho_{t}(u)}\right)^{o}$, then for all $\gamma \in\left(0, \gamma_{t}\right)$ the function $\gamma G_{t}$ satisfies the assumptions of Lemma 4.4, and thus for $\gamma \in\left(0, \gamma_{t}\right)$ the term in (51) is bounded above by

$$
\int_{\mathbb{T}^{d}} \sup _{\lambda \in \mathbb{R}_{+}^{2}}\left\{\gamma G_{t}(u, \lambda)-\frac{1}{2} \Lambda_{\rho_{t}(u)}^{*}(\lambda)\right\} d u .
$$

To complete the application of the relative entropy method, it remains to show that by reducing $\gamma_{t}>0, t \in\left[0, T_{\max }\right)$, if necessary, this last term is non-positive.

We note that this would follow if we had a bound of the form

$$
B_{t}:=\sup _{\substack{\rho \in K_{t} \\ \lambda \in \mathbb{R}_{+}^{2}}} \frac{|\Psi(\rho, \lambda)|}{\Lambda_{\rho}^{*}(\lambda)}<+\infty
$$

where $K_{t} \subseteq A:=\boldsymbol{R}\left(\mathcal{D}_{\boldsymbol{R}}^{o}\right) \cap(0, \infty)^{2}$ is a compact set containing the image $\boldsymbol{\rho}_{t}\left(\mathbb{T}^{d}\right)$. Indeed, since $\Lambda_{\rho}^{*}(\lambda)=0$ iff $\lambda=\rho$, in which case $\Psi(\rho, \lambda)=0$, we would then have that

$$
|\Psi(\rho, \lambda)| \leq B_{t} \Lambda_{\rho}^{*}(\lambda) \quad \text { for all }(\rho, \lambda) \in K_{t} \times \mathbb{R}_{+}^{2},
$$

and so for $\gamma \in\left(0, \gamma_{t}\right)$ we would have

$$
\gamma\left|G_{t}(u, \lambda)\right| \leq \gamma\left\|\frac{\Delta\left[\boldsymbol{\Phi}\left(\rho_{t}\right)\right]}{\boldsymbol{\Phi}\left(\boldsymbol{\rho}_{t}\right)}\right\|_{L^{\infty}\left(\mathbb{T}^{d} ; \ell_{\infty}^{2}\right)} B_{t} \Lambda_{\rho_{t}(u)}^{*}(\lambda)
$$

for all $(u, \lambda) \in \mathbb{T}^{d} \times \mathbb{R}_{+}^{2}$. Then by choosing $\gamma_{t}>0$ small enough so that in addition $\gamma_{t} B_{t}\left\|\Delta\left[\boldsymbol{\Phi}\left(\rho_{t}\right)\right] / \boldsymbol{\Phi}\left(\rho_{t}\right)\right\|_{L^{\infty}\left(\mathbb{T}^{d} ; \ell_{\infty}^{2}\right)}<\frac{1}{2}$, it would follow that (56) is non-positive, and the proof would be complete. The bound (57) is proved in Lemma 4.5. Before we proceed with the proof of Lemma 4.5, we recall some facts on recession functions of convex functions.

Given a lower semicontinuous proper convex function $\psi: \mathbb{R}^{d} \rightarrow(-\infty,+\infty]$ with $0 \in$ $\mathcal{D}_{\psi}$, its recession function $\psi_{\infty}: \mathbb{R}^{d} \rightarrow(-\infty,+\infty]$ is defined by

$$
\psi_{\infty}(y):=\lim _{t \rightarrow+\infty} \frac{\psi(t y)}{t}=\left.\lim _{t \rightarrow+\infty} \frac{d}{d t}\right|_{+} \psi(t y),
$$

where $\left.\frac{d}{d t}\right|_{+}$denotes differentiation from the right. The recession function $\psi_{\infty}$ is obviously positively 1-homogeneous, $\psi_{\infty}(\lambda y)=\lambda \psi_{\infty}(y)$ for all $y \in \mathbb{R}^{d}, \lambda \geq 0$. 
It is well known [22, Theorem 8.5] that if $\psi$ is a proper lower semi-continuous convex function, then so is its recession function. Using the equivalent definition of recession functions via the recession cone of their epigraphs [22, Sect. 8], one can express the recession function by the formula

$$
\psi_{\infty}(y)=\inf \left\{\liminf _{k \rightarrow+\infty} \frac{\psi\left(t_{k} y_{k}\right)}{t_{k}} \mid t_{k} \rightarrow+\infty, y_{k} \rightarrow y\right\}
$$

(see $[10,(12.7 .1)])$. Particularly useful in the proof of the following lemma is the characterisation of the interior of the proper domain of a convex function $\psi$ via the recession function of its Legendre transform, as stated in [10, (12.7.3)],

$$
\mathcal{D}_{\psi}^{o}=\bigcap_{y \neq 0}\left\{x \in \mathbb{R}^{d} \mid\langle x, y\rangle<\left(\psi^{*}\right)_{\infty}(y)\right\} .
$$

Applying (59) to the thermodynamic pressure $P:=\log \mathcal{Z}, \mathcal{Z}:=Z \circ$ exp, we get

$$
\log \left(\mathcal{D}_{Z}^{o} \cap(0, \infty)^{2}\right)=\mathcal{D}_{\mathcal{Z}}^{o}=\left\{\boldsymbol{\mu} \in \mathbb{R}^{2} \mid S_{\infty}(\lambda)>\langle\lambda, \boldsymbol{\mu}\rangle, \forall \lambda \neq 0\right\} .
$$

In other words, $\mathcal{D}_{\mathcal{Z}}^{o}$ is the intersection of all hyperplanes $\left\{\boldsymbol{\mu} \in \mathbb{R}^{2} \mid\langle\boldsymbol{\mu}, \boldsymbol{v}\rangle<S_{\infty}(\boldsymbol{v})\right\}$ for $\boldsymbol{v} \in S^{1} \cap(0, \infty)^{2}$. This implies that the function $S^{1} \cap(0, \infty)^{2} \ni \boldsymbol{v} \mapsto S_{\infty}(\boldsymbol{v}) \boldsymbol{v} \in \mathbb{R}^{2}$ is a parametrisation of the boundary $\partial \mathcal{D}_{\mathcal{Z}}$. This may be compared with [13, (2.14)]. Consequently, the part of the boundary $\partial \mathcal{D}_{Z}$ on the strictly positive quadrant is given by the parametrisation $e^{S_{\infty}(\boldsymbol{v}) \boldsymbol{v}}, \boldsymbol{v} \in S^{1} \cap(0, \infty)^{2}$. Along the two axes $\varphi_{1}=0$ and $\varphi_{2}=0$, there is only one-species of particles and the critical fugacities in these directions are fugacities of one species ZRPs.

Lemma 4.5 For any compact $K \subseteq A:=\boldsymbol{R}\left(\mathcal{D}_{\boldsymbol{R}}^{o}\right) \cap(0, \infty)^{2}$,

$$
\sup _{(\rho, \lambda) \in K \times \mathbb{R}_{+}^{2}} \frac{|\Psi(\rho, \lambda)|}{\Lambda_{\rho}^{*}(\lambda)}<+\infty .
$$

Proof For all $(\rho, \lambda) \in K \times(0, \infty)^{2}$ we have that $\Lambda^{*}(\rho, \lambda):=\Lambda_{\rho}^{*}(\lambda) \geq 0$ and the functions $|\Psi|: K \times(0, \infty)^{2} \rightarrow \mathbb{R}_{+}$and $\Lambda^{*}: K \times(0, \infty)^{2} \rightarrow \mathbb{R}_{+}$are continuous. Therefore the fraction in the supremum can tend to infinity if the nominator goes to infinity or the denominator goes to zero. Since $\boldsymbol{\Psi}: K \times(0, \infty)^{2} \rightarrow \mathbb{R}^{2}$ is continuous and $K$ is compact the nominator can tend to infinity only as $|\lambda|_{1} \rightarrow+\infty$. In this case $\Lambda_{\rho}^{*}$ also tends to $+\infty$ as a rate functional with compact level sets. Since $\Lambda_{\rho}^{*}$ is the rate functional of the i.i.d. occupation variables $\eta(x)$, $x \in \mathbb{Z}^{d}$, with common law $v_{\rho}^{1}$ we have that $\Lambda^{*}(\rho, \lambda)=0$ iff $\rho=\lambda$ for the denominator. But obviously for $\rho=\lambda$ we have $\boldsymbol{\Psi}(\boldsymbol{\rho}, \lambda)=0$, so the nominator vanishes as well. So in order to prove the lemma we have to show that the nominator and the denominator are of the same order as $|\rho-\lambda| \rightarrow 0$ and $|\rho-\lambda| \rightarrow+\infty$.

Motivated by the previous sketch, we choose $\varepsilon>0$ such that $K_{\varepsilon}:=\overline{K^{(\varepsilon)}} \subseteq A$, where $K^{(\varepsilon)}:=\bigcup_{x \in K} D(x, \varepsilon)$, and for any $M>0$ we separate the region $K \times(0, \infty)^{2}$ as $K \times$ $(0, \infty)^{2}=\mathcal{E}_{0}^{\varepsilon} \cup \mathcal{E}_{\varepsilon}^{M} \cup \mathcal{E}_{M}^{\infty}$, where

$$
\begin{aligned}
\mathcal{E}_{0}^{\varepsilon} & :=\left\{(\rho, \lambda) \in K \times(0, \infty)^{2}|| \rho-\lambda \mid \leq \varepsilon\right\}, \\
\mathcal{E}_{\varepsilon}^{M} & :=\left\{(\rho, \lambda) \in K \times(0, \infty)^{2}|\varepsilon \leq| \rho-\lambda \mid \leq M\right\}, \\
\mathcal{E}_{M}^{\infty} & :=\left\{(\rho, \lambda) \in K \times(0, \infty)^{2}|| \rho-\lambda \mid \geq M\right\} .
\end{aligned}
$$

We prove the claim on each region individually. Obviously the set $\mathcal{E}_{\varepsilon}^{M}$ is compact and so since the functions $\boldsymbol{\Psi}$ and $\Lambda^{*}$ are jointly continuous, the claim holds on the region $\mathcal{E}_{\varepsilon}^{M}$. 
We turn to the region $\mathcal{E}_{0}^{\varepsilon}$. By its definition, for any $(\rho, \lambda) \in \mathcal{E}_{0}^{\varepsilon}$ we have that $\lambda \in D(\rho, \varepsilon) \subseteq$ $K_{\varepsilon} \subseteq A$. So, since $D(\rho, \varepsilon)$ is convex, for all $(\rho, \lambda) \in \mathcal{E}_{0}^{\varepsilon}$ the image of the constant speed line segment $\gamma_{\rho, \lambda}:[0,1] \rightarrow \mathbb{R}^{2}$ from $\rho$ to $\lambda$ is contained in $K_{\varepsilon}$, i.e.,

$$
\gamma_{\rho, \lambda}([0,1]) \subseteq K_{\varepsilon} \quad \text { for all }(\rho, \lambda) \in \mathcal{E}_{0}^{\varepsilon} .
$$

By the first order Taylor expansion of $\Phi_{i}, i=1,2$ around the point $\rho \in K$,

$$
\Psi_{i}(\rho, \lambda)=\int_{0}^{1}(1-t)\left\langle\lambda-\rho, D^{2} \Phi_{i}\left(\gamma_{\rho, \lambda}(t)\right)(\lambda-\rho)\right\rangle d t \quad \text { for all }(\rho, \lambda) \in \mathcal{E}_{0}^{\varepsilon} .
$$

Since $\boldsymbol{\Phi}$ is smooth on the set $A$, the matrix $D^{2} \Phi_{i}(\rho)$ is symmetric for all $\rho \in A$. Denoting by $\lambda_{ \pm}^{i}(\rho)$ the real eigenvalues of $D^{2} \Phi_{i}(\rho)$ we have

$$
\lambda_{-}^{i}\left(\gamma_{\rho, \lambda}(t)\right)|\lambda-\rho|^{2} \leq\left\langle\lambda-\rho, D^{2} \Phi_{i}\left(\gamma_{\rho, \lambda}(t)\right)(\lambda-\rho)\right\rangle \leq \lambda_{+}^{i}\left(\gamma_{\rho, \lambda}(t)\right)|\lambda-\rho|^{2} .
$$

Furthermore, by the continuity of the eigenvalues $\lambda_{ \pm}^{i}$ as functions of $\rho \in A$,

$$
A^{i}:=\sup _{\rho \in K_{\varepsilon}}\left|\lambda_{-}^{i}\right| \vee\left|\lambda_{+}^{i}\right|(\rho)<+\infty .
$$

So by (62), we have that $\left|\lambda_{-}^{i}\right| \vee\left|\lambda_{+}^{i}\right|\left(\gamma_{\rho, \lambda}(t)\right) \leq A^{i}$ for all $(t, \rho, \lambda) \in[0,1] \times \mathcal{E}_{0}^{\varepsilon}$ and thus

$$
\left|\Psi_{i}(\rho, \lambda)\right| \leq \frac{A^{i}}{2}|\lambda-\rho|^{2}, \quad i=1,2 .
$$

For the denominator in (61), we note that the rate functional $\Lambda_{\rho}^{*}$ is $C^{1}$ on $(0, \infty)^{2}$ and $C^{2}$ on $A$ with

$$
\begin{gathered}
\nabla \Lambda_{\rho}^{*}(\lambda)=\log \frac{\overline{\boldsymbol{\Phi}}(\lambda)}{\boldsymbol{\Phi}(\rho)}, \quad \lambda \in(0, \infty)^{2}, \\
D^{2} \Lambda_{\rho}^{*}(\lambda)=D \log \boldsymbol{\Phi}(\boldsymbol{\lambda})=D^{2} S(\lambda), \quad \lambda \in A,
\end{gathered}
$$

where $S$ is the thermodynamic entropy. Since $\Lambda_{\rho}^{*}$ and its derivative vanish at $\rho$, by Taylor expansion of $\Lambda_{\rho}^{*}$ around $\rho \in K$

$$
\Lambda_{\rho}^{*}(\lambda)=\int_{0}^{1}(1-t)\left\langle\lambda-\rho, D^{2} S\left(\gamma_{\rho, \lambda}(t)\right)(\lambda-\rho)\right\rangle d t, \quad \lambda \in A .
$$

Denoting by $\lambda_{-}(\rho)>0$ the minimal eigenvalue of the strictly positive definite matrix $D^{2} S(\rho)$, we have by continuity that

$$
B:=\inf _{\rho \in K_{\varepsilon}} \lambda_{-}(\rho)>0 .
$$

Then $\Lambda_{\rho}^{*}(\lambda) \geq \frac{B}{2}|\lambda-\rho|^{2}$ for all $(\rho, \lambda) \in \mathcal{E}_{0}^{\varepsilon}$, which shows that

$$
\sup _{(\rho, \lambda) \in \mathcal{E}_{0}^{\varepsilon}} \frac{\left|\Psi_{i}(\rho, \lambda)\right|}{\Lambda_{\rho}(\lambda)} \leq \frac{A^{i}}{B}<+\infty, \quad i=1,2
$$

and yields the bound (61) in the region $\mathcal{E}_{0}^{\mathcal{E}}$.

It remains to show that the supremum is finite in the region $\mathcal{E}_{M}^{\infty}$ for some $M>0$. On one hand, it follows from (16) and the compactness of $K$ that $\boldsymbol{\Psi}$ satisfies a bound of the form

$$
|\Psi(\rho, \lambda)|_{1} \leq C_{0}+C_{1}|\lambda|_{1} \quad \forall(\rho, \lambda) \in K \times \mathbb{R}_{+}^{2}
$$


for some constants $C_{0}, C_{1} \geq 0$. So, to complete the proof, it suffices to show that $\Lambda^{*}$ has at least linear growth in $\mathcal{E}_{M}^{\infty}$ as $|\lambda| \rightarrow+\infty$, i.e.,

$$
\lim _{M \rightarrow+\infty} \inf _{(\rho, \lambda) \in \mathcal{E}_{M}^{\infty}} \frac{\Lambda_{\rho}^{*}(\lambda)}{|\lambda|}>0,
$$

where of course the limit as $M \rightarrow+\infty$ exists as an increasing limit. We begin by noting that

$$
\lim _{M \rightarrow+\infty} \inf _{(\rho, \lambda) \in \mathcal{E}_{M}^{\infty}} \frac{\Lambda_{\rho}^{*}(\lambda)}{|\lambda|} \geq \liminf _{|\lambda| \rightarrow+\infty} \inf _{\rho \in K} \frac{\Lambda_{\rho}^{*}(\lambda)}{|\lambda|}=: a .
$$

We choose a sequence $\left\{\lambda_{n}\right\} \subseteq \mathbb{R}_{+}^{2}$ achieving the limit inferior,

$$
\left|\lambda_{n}\right| \rightarrow+\infty \text { and } \lim _{n \rightarrow+\infty} \inf _{\rho \in K} \frac{\Lambda_{\rho}^{*}\left(\lambda_{n}\right)}{\left|\lambda_{n}\right|}=a .
$$

Since $\left\{\frac{\lambda_{n}}{\left|\lambda_{n}\right|}\right\}$ is contained in the compact space $S_{+}^{1}:=S^{1} \cap \mathbb{R}_{+}^{2}$, by passing to a subsequence if necessary, we can assume that $\left\{\frac{\lambda_{n}}{\left|\lambda_{n}\right|}\right\}$ converges to some direction $\boldsymbol{v} \in S_{+}^{1}$. Then obviously

$$
\liminf _{n \rightarrow+\infty} \frac{S\left(\lambda_{n}\right)}{\left|\lambda_{n}\right|} \geq \liminf _{\substack{|\lambda| \rightarrow+\infty \\ \lambda /|\lambda| \rightarrow v}} \frac{S(\lambda)}{|\lambda|}=S_{\infty}(v),
$$

where the equality in the right-hand side holds by (58). Since $\Phi(K) \subseteq \mathcal{D}_{Z}^{o} \cap(0, \infty)^{2}$, we have by (60) that $S_{\infty}(\boldsymbol{v})-\langle\boldsymbol{v}, \log \boldsymbol{\Phi}(\boldsymbol{\rho})\rangle>0$ for all $\rho \in K$. Thus, since $\boldsymbol{\Phi}$ is continuous and $K$ is compact,

$$
\theta:=\inf _{\boldsymbol{\rho} \in K}\left\{S_{\infty}(\boldsymbol{v})-\langle\boldsymbol{v}, \log \boldsymbol{\Phi}(\boldsymbol{\rho})\}>0 .\right.
$$

Then by (63) there exists $n_{1} \in \mathbb{N}$ such that

$$
n \geq n_{1} \Longrightarrow \frac{S\left(\lambda_{n}\right)}{\left|\lambda_{n}\right|} \geq S_{\infty}(v)-\frac{\theta}{3} .
$$

By (54) and taking into account the fact that $Z \geq 1$, we have that for all $n \geq n_{1}$ and all $\rho \in K$,

$$
\begin{aligned}
\frac{\Lambda_{\boldsymbol{\rho}}^{*}\left(\lambda_{n}\right)}{\left|\lambda_{n}\right|} & \geq S_{\infty}(\boldsymbol{v})-\left\langle\frac{\lambda_{n}}{\left|\lambda_{n}\right|}, \log \boldsymbol{\Phi}(\boldsymbol{\rho})\right\rangle+\frac{1}{\left|\lambda_{n}\right|} \log Z(\boldsymbol{\Phi}(\boldsymbol{\rho}))-\frac{\theta}{3} \\
& \geq S_{\infty}(\boldsymbol{v})-\left\langle\frac{\lambda_{n}}{\left|\lambda_{n}\right|}, \log \boldsymbol{\Phi}(\boldsymbol{\rho})\right\rangle-\frac{\theta}{3} .
\end{aligned}
$$

But by the compactness of $K$, we have that $\|\log \boldsymbol{\Phi}\|_{L^{\infty}(K)}:=\sup _{\rho \in K}|\log \boldsymbol{\Phi}(\rho)|_{2}<+\infty$ and therefore the sequence $\left\{\left\langle\frac{\lambda_{n}}{\left|\lambda_{n}\right|}, \log \Phi(\rho)\right\rangle\right\}$ converges to $\langle\boldsymbol{v}, \log \Phi(\rho)\rangle$ uniformly over all $\rho \in K$,

$$
\sup _{\rho \in K}\left|\left\langle\frac{\lambda_{n}}{\left|\lambda_{n}\right|}, \log \boldsymbol{\Phi}(\boldsymbol{\rho})\right\rangle-\langle\boldsymbol{v}, \log \boldsymbol{\Phi}(\boldsymbol{\rho})\rangle\right| \leq\|\log \boldsymbol{\Phi}\|_{L^{\infty}(K)}\left|\frac{\lambda_{n}}{\left|\lambda_{n}\right|}-\boldsymbol{v}\right|_{2} \rightarrow 0 .
$$

Therefore we can choose $n_{2} \in \mathbb{N}$ such that

$$
n \geq n_{2} \Longrightarrow \sup _{\boldsymbol{\rho} \in K}\left|\left\langle\frac{\lambda_{n}}{\left|\lambda_{n}\right|}, \log \boldsymbol{\Phi}(\boldsymbol{\rho})\right\rangle-\langle\boldsymbol{v}, \log \boldsymbol{\Phi}(\boldsymbol{\rho})\rangle\right|<\frac{\theta}{3},
$$


and then for all $n \geq n_{1} \vee n_{2}$ and all $\rho \in K$

$$
\frac{\Lambda_{\rho}^{*}\left(\lambda_{n}\right)}{\left|\lambda_{n}\right|} \geq S_{\infty}(\boldsymbol{v})-\langle\boldsymbol{v}, \log \boldsymbol{\Phi}(\boldsymbol{\rho})\rangle-\frac{2 \theta}{3} \geq \theta-\frac{2 \theta}{3}=\frac{\theta}{3}>0 .
$$

This proves that

$$
\liminf _{|\lambda| \rightarrow+\infty} \inf _{\rho \in K} \frac{\Lambda_{\rho}^{*}(\lambda)}{|\lambda|}=\lim _{n \rightarrow+\infty} \inf _{\rho \in K} \frac{\Lambda_{\rho}^{*}\left(\lambda_{n}\right)}{\left|\lambda_{n}\right|}>0
$$

which completes the proof of Lemma 4.5.

Since Lemma 4.5 establishes the missing bound (57), the proof of Theorem 3.2 is complete.

\subsection{Proof of Theorem 3.3}

By [1], it is known that quasilinear parabolic systems have unique maximal classical solutions when starting from initial profiles of class $C^{2+\theta}, \theta \in[0,1)$. To show that classical solutions of the species-blind parabolic system (26) are global in time, we prove first a maximum principle asserting that the region

$$
A:=\boldsymbol{R}\left(\mathcal{D}_{\boldsymbol{R}}^{o}\right) \cap(0, \infty)^{2}=\left\{\rho \in(0, \infty)^{2} \mid \rho_{1}+\rho_{2}<\hat{\rho}_{c}\right\}
$$

is invariant under the evolution of the species-blind parabolic system. Here $\hat{\rho}_{c} \in(0,+\infty]$ is the critical density of the one-species ZRP associated with the species-blind ZRP. The proof of this version of the maximum principle for systems of the form (26) relies on the maximum principle for quasilinear PDEs in divergence form found in [2]. Since, as we will see, the solution $\rho$ cannot lose regularity, we will obtain the existence of global in time classical solutions.

Lemma 4.6 (A maximum principle for the species-blind parabolic system) Let $\rho=$ $\left(\rho_{1}, \rho_{2}\right) \in C^{1,2}\left([0, T) \times \mathbb{T}^{d} ; \mathbb{R}^{2}\right), T>0$, be a classical solution of the species-blind parabolic system (26) starting from an initial condition $\rho_{0} \in C^{2}\left(\mathbb{T}^{d} ; \mathbb{R}_{+}^{2}\right)$ satisfying

$$
\rho_{0}\left(\mathbb{T}^{d}\right) \subseteq A=\left\{\rho \in(0, \infty)^{2} \mid \rho_{1}+\rho_{2}<\hat{\rho}_{c}\right\},
$$

where $\hat{\rho}_{c}$ is the critical density corresponding to the one-species density function $\hat{R}$. Then

$$
\begin{aligned}
0 & <\inf _{(t, u) \in[0 ; T) \times \mathbb{T}^{d}} \rho_{1}(t, u) \wedge \rho_{2}(t, u) \\
& \leq \sup _{(t, u) \in[0, T) \times \mathbb{T}^{d}}\left(\rho_{1}(t, u)+\rho_{2}(t, u)\right) \leq \sup _{u \in \mathbb{T}^{d}}\left(\rho_{1}(0, u)+\rho_{2}(0, u)\right)<\hat{\rho}_{c} .
\end{aligned}
$$

Proof By the continuity of $\rho_{0}$ and the compactness of $\mathbb{T}^{d}$, there exists by assumption (64) an $\varepsilon>0$ such that

$$
\rho_{0}\left(\mathbb{T}^{d}\right) \subseteq\left\{\left(\rho_{1}, \rho_{2}\right) \in \mathbb{R}^{2} \mid \rho_{1} \wedge \rho_{2}>\varepsilon, \rho_{1}+\rho_{2}<\hat{\rho}_{c}-\varepsilon\right\},
$$

where we replace $\hat{\rho}_{c}-\varepsilon$ by $\frac{1}{\varepsilon}$ when $\hat{\rho}_{c}=+\infty$. Since $\rho$ solves (26), by summing the two equations we see that the function $\rho_{1}+\rho_{2}$ solves the equation $\partial_{t} \rho=\Delta \hat{\Phi}(\rho)$. But since $\hat{\Phi}$ is the mean jump rate of a single species ZRP,

$$
0<c<\hat{\Phi}^{\prime}(\rho)<C<+\infty \text { for all } \rho \in\left[0, \hat{\rho}_{c}-\varepsilon / 2\right]
$$


for some constants $c, C \geq 0$ and therefore the equation $\partial_{t} \rho=\Delta \hat{\Phi}(\rho)$ is uniformly parabolic, when considered for sub-critical initial conditions $\rho_{0} \in C\left(\mathbb{T}^{d},\left(0, \hat{\rho}_{c}\right)\right)$. Therefore it follows by (66) and the maximum principle for scalar uniformly parabolic quasilinear equations that

$$
2 \varepsilon<\inf _{(t, u) \in[0 ; T) \times \mathbb{T}^{d}}\left(\rho_{1}+\rho_{2}\right)(t, u) \leq \sup _{(t, u) \in[0 ; T) \times \mathbb{T}^{d}}\left(\rho_{1}+\rho_{2}\right)(t, u)<\hat{\rho}_{c}-\varepsilon .
$$

We consider now the family of the open domains

$$
B_{\delta}:=\left\{\left(\rho_{1}, \rho_{2}\right) \in \mathbb{R}^{2} \mid \varepsilon<\rho_{1}+\rho_{2}<\hat{\rho}_{c}-\varepsilon, \rho_{1} \wedge \rho_{2}>-\delta\right\}
$$

for $\delta \in[0,+\infty]$ and set

$$
D_{\delta}:=\left\{(t, u, r) \in[0, T) \times \mathbb{T}^{d} \times \mathbb{R} \mid\left(r, \rho_{2}(t, u)\right) \in B_{\delta}\right\} .
$$

Let $\Psi: D_{\infty} \rightarrow \mathbb{R}$ denote the function given by the formula

$$
\Psi(t, u, r)=r \frac{\hat{\Phi}\left(r+\rho_{2}(t, u)\right)}{r+\rho_{2}(t, u)} .
$$

The sets $D_{\delta}$ are obviously open and the function $\Psi$ is well defined on $D_{\infty}$. Since the sum $\rho_{1}+\rho_{2}$ satisfies (68), we have that

$$
\left(t, u, \rho_{1}(t, u)\right) \in D_{\infty} \text { for all }(t, u) \in[0, T) \times \mathbb{T}^{d},
$$

and since $\left(\rho_{1}, \rho_{2}\right)$ is a solution of $(26)$, we have that $\rho_{1}$ solves

$$
\partial_{t} \rho_{1}(t, u)=\Delta \Psi\left(t, u, \rho_{1}(t, u)\right) .
$$

In divergence form, the problem above is written as

$$
\partial_{t} \rho_{1}(t, u)=\operatorname{div} A_{\Psi}\left(t, u, \rho_{1}(t, u), \nabla \rho_{1}(t, u)\right)
$$

where $A_{\Psi}: D_{\infty} \rightarrow \mathbb{R}^{d}$ is the function given by the formula

$$
A_{\Psi}(t, u, r, v)=\nabla_{u} \Psi(t, u, r)+\partial_{r} \Psi(t, u, r) v .
$$

Since $\rho_{2}$ is $C^{1,2}$, it follows that the function $A_{\Psi}$ is $C^{1}$ and $\partial_{v} A_{\Psi}(t, u, r, v)=\partial_{r} \Psi(t, u, r) I$ where $I \in \mathbb{R}^{d \times d}$ denotes the identity matrix. By a simple calculation, $\partial_{r} \Psi(t, u, r)=$ $H\left(r, \rho_{2}(t, u)\right)$, where $H: B_{\infty} \rightarrow \mathbb{R}$ is given by

$$
H\left(\rho_{1}, \rho_{2}\right)=\frac{\rho_{2}}{\rho_{1}+\rho_{2}} \frac{\hat{\Phi}\left(\rho_{1}+\rho_{2}\right)}{\rho_{1}+\rho_{2}}+\frac{\rho_{1}}{\rho_{1}+\rho_{2}} \hat{\Phi}^{\prime}\left(\rho_{1}+\rho_{2}\right) .
$$

We have that

$$
\inf _{B_{\delta}} H \leq \inf _{D_{\delta}} \partial_{r} \Psi \leq \sup _{D_{\delta}} \partial_{r} \Psi \leq \sup _{B_{\delta}} H
$$

for all $\delta \in[0,+\infty]$ and it is obvious that

$$
c \leq \inf _{B_{0}} H \leq \sup _{B_{0}} H \leq C,
$$

where $c, C \geq 0$ are the constants in (67). By continuity of $H$, we obtain the existence of $\delta_{0}>0$ such that

$$
\frac{c}{2}<\inf _{B_{\delta_{0}}} H \leq \sup _{B_{\delta_{0}}} H<2 C,
$$


which shows that the diagonal matrix $\partial_{v} A_{\Psi}$ is positive definite on the set $D_{\delta_{0}} \times \mathbb{R}^{d}$. We set now

$$
T^{i}:=\sup \left\{t \in[0, T] \mid \inf _{(s, u) \in[0, t) \times \mathbb{T}^{d}} \rho_{i}(s, u)>0\right\}, \quad i=1,2 .
$$

By the assumptions on the initial condition $\boldsymbol{\rho}_{0}$, the set over which we take the supremum is non-empty. By the continuity of the solution $\rho$, we have $T^{i}>0$ for $i=1,2$ and if $T^{i}<T$ then there exists $u_{0}^{i} \in \mathbb{T}^{d}$ such that $\rho_{i}\left(T^{i}, u_{0}^{i}\right)=0$. In order to prove the claim of the lemma, it suffices to show that $T^{1}=T^{2}=T$.

So we suppose that this is not true to obtain a contradiction. Without loss of generality it suffices to consider the cases $T^{1}<T^{2}<T$ and $T^{0}:=T^{1}=T^{2}<T$.

(a) $T^{1}<T^{2}<T$ : Since $\rho_{1}(t, u) \geq 0$ for all $(t, u) \in\left[0, T^{1}\right] \times \mathbb{T}^{d}$ and $\rho_{1}$ is continuous in $[0, T) \times \mathbb{T}^{d}$, there exists $t_{0}>0$ such that

$$
\inf _{(t, u) \in\left[0, T^{1}+t_{0}\right] \times \mathbb{T}^{d}} \rho_{1}(t, u)>-\delta_{0} .
$$

But then $\left(t, u, \rho_{1}(t ; u)\right) \in D_{\delta_{0}}$ for all $(t, u) \in\left[0, T^{1}+t_{0}\right] \times \mathbb{T}^{d}$ and so, since $\rho_{1}$ and 0 are solutions of problem (69) in $\left[0, T^{1}+t_{0}\right] \times \mathbb{T}^{d}$, which is uniformly parabolic in this region by (70) and (71), and since $\rho_{1}\left(T^{1}, u_{0}^{1}\right)=0$, we get from [2, Theorem 1] that $\rho_{1} \equiv 0$ in $\left[0, T^{1}\right) \times \mathbb{T}^{d}$, which contradicts the definition of $T^{1}$.

(b) $T^{0}:=T^{1}=T^{2}<T$ : Again, since $\rho_{1}(t, u) \wedge \rho_{2}(t, u)>0$ for all $(t, u) \in\left[0, T^{0}\right] \times \mathbb{T}^{d}$, there exists $t_{0}>0$ such that

$$
\inf _{(t, u) \in\left[0, T^{1}+t_{0}\right] \times \mathbb{T}^{d}}\left[\rho_{1}(t, u) \wedge \rho_{2}(t, u)\right] \geq-\delta_{0} .
$$

But then again the problem (69) is uniformly parabolic in $\left[0, T^{0}+t_{0}\right] \times \mathbb{T}^{d}$ and $\rho_{1}$ and 0 are solutions with $\rho_{1} \geq 0$ in $\left[0, T^{0}\right]$, which again by [2, Theorem 1$]$ yields $\rho_{1} \equiv 0$ in $\left[0, T^{0}\right) \times \mathbb{T}^{d}$ and contradicts the definition of $T^{0}$.

Using this maximum principle and the global existence of scalar uniformly parabolic equations, we obtain the global existence of solutions to the species-blind parabolic system as follows. To derive a contradiction, we assume that $\rho \in C^{1,2}\left(\left[0, T_{\max }\right) \times \mathbb{T}^{d} ; \mathbb{R}^{2}\right), T_{\max }<$ $+\infty$, is the maximal classical solution of the species-blind parabolic system starting from $\rho_{0} \in C^{2+\theta}\left(\mathbb{T}^{d}\right)$. Here maximality of the solution means that $\rho$ can not be extended to a $C^{1,2}$ solution on $[0, T) \times \mathbb{T}^{d}$ for $T>T_{\max }$. Since $\hat{\rho}_{0}:=\rho_{01}+\rho_{02} \in C^{1+\theta ; 2+\theta}\left(\mathbb{T}^{d} ;\left(0, \rho_{c}\right)\right)$, there exists a unique solution $\hat{\rho} \in C^{1+\theta, 2+\theta}\left(\mathbb{R}_{+} \times \mathbb{T}^{d} ;\left(0, \rho_{c}\right)\right)$ of the scalar quasilinear parabolic equation $\partial_{t} \rho=\Delta \hat{\Phi}(\rho)$ with initial data $\hat{\rho}_{0}$. Then, $\hat{\rho}\left(\mathbb{R}_{+} \times \mathbb{T}^{d}\right) \subseteq\left(\varepsilon, \hat{\rho}_{c}-\varepsilon\right)$ for some $\varepsilon>0$ and the function $\phi(x):=\frac{\hat{\Phi}(x)}{x}$ is $C^{\infty}$ in $\left[\varepsilon, \hat{\rho}_{c}-\varepsilon\right]$. Thus the function $a: \mathbb{R}_{+} \times \mathbb{T}^{d} \rightarrow \mathbb{R}_{+}$ defined by $a(t, u):=\frac{\hat{\Phi}(\hat{\rho}(t, u))}{\hat{\rho}(t, u)}$ belongs to $C^{1+\theta, 2+\theta}\left(\mathbb{R}_{+} \times \mathbb{T}^{d}\right)$. Since $\hat{\Phi}^{\prime}$ satisfies (67),

$$
0<c<a(t, u) \leq C<+\infty \text { for all }(t, u) \in \mathbb{R}_{+} \times \mathbb{T}^{d}
$$

for some constants $c, C \geq 0$. Since the function $\rho_{1}+\rho_{2}$ is also a solution of the scalar equation $\partial_{t} \rho=\Delta \hat{\Phi}(\rho)$ with the same initial data $\rho_{0}$, we have by the uniqueness of solutions that

$$
a \equiv \frac{\hat{\Phi}\left(\rho_{1}+\rho_{2}\right)}{\rho_{1}+\rho_{2}} \quad \text { in }\left[0, T_{\max }\right) \times \mathbb{T}^{d} .
$$


We consider the system

$$
\left\{\begin{array}{l}
\partial_{t} \rho_{1}=\Delta\left(a(t, u) \rho_{1}(t, u)\right) \\
\partial_{t} \rho_{2}=\Delta\left(a(t, u) \rho_{2}(t, u)\right)
\end{array} \quad, \quad \rho(0, \cdot)=\left(\rho_{01}, \rho_{02}\right) \quad \text { in } \mathbb{T}^{d},\right.
$$

which is obviously decoupled and can be solved by solving the scalar linear second order parabolic equation

$$
\partial_{t} \rho=\Delta(a(t, u) \rho(t, u))
$$

twice with initial conditions $\rho_{01}$ and $\rho_{02}$. This scalar equation is given in general form by

$$
\partial_{t} \rho=\sum_{i, j=1}^{d} a^{i j} \partial_{i j}^{2} \rho+\sum_{i=1}^{d} b^{i} \partial_{i} \rho+c \rho,
$$

where $a^{i j}=a \delta_{i j}, b^{i}=\partial_{i} a$ and $c=\Delta a$. We note that since $a$ satisfies (72) and $a^{i j}=a \delta_{i j}$, the matrix $\left(a^{i j}\right)$ is uniformly elliptic. Also, since $a \in C^{1+\theta, 2+\theta}\left(\mathbb{R}_{+} \times \mathbb{T}^{d}\right)$, the coefficients $a^{i j}, b^{i}, c$ are $\theta$-Hölder continuous and so by the interpretation of [20, Theorem 5.14] in the flat torus with periodic boundary conditions, we find that for any $\rho_{0} \in C^{2+\theta}\left(\mathbb{T}^{d}\right)$ there exists a unique solution $\rho \in C_{\text {loc }}^{1+\theta, 2+\theta}\left(\mathbb{R}_{+} \times \mathbb{T}^{d}\right)$ to the scalar problem (75) with initial condition $\rho_{0}$, and thus there exists a unique solution $\tilde{\boldsymbol{\rho}} \in C_{\mathrm{loc}}^{1+\theta, 2+\theta}\left(\mathbb{R}_{+} \times \mathbb{T}^{d} ; \mathbb{R}^{2}\right)$ of system (74) starting from $\rho_{0}=\left(\rho_{01}, \rho_{02}\right)$. Since by (73), we have that the solution $\rho \in C^{1,2}\left(\left[0, T_{\max }\right) \times \mathbb{T}^{d} ; \mathbb{R}^{2}\right)$ of the system (26) also solves the system (74), it follows by the uniqueness of solutions that $\tilde{\rho}=\rho$ in $\left[0, T_{\max }\right) \times \mathbb{T}^{d}$. This, taking also into account the maximum principle, shows that

$$
\rho \in C^{1+\theta, 2+\theta}\left(\left[0, T_{\max }\right) \times \mathbb{T}^{d} ; A\right) .
$$

Now, we obviously have that $\tilde{\boldsymbol{\rho}}_{T_{\max }} \in C^{2+\theta}\left(\mathbb{T}^{d}\right)$, and since $\tilde{\boldsymbol{\rho}}$ solves (26) in $\left[0, T_{\max }\right) \times \mathbb{T}^{d}$, we have by the maximum principle that

$$
\tilde{\boldsymbol{\rho}}\left(\left[0, T_{\max }\right) \times \mathbb{T}^{d}\right) \subseteq\{\boldsymbol{r} \in A \mid d(\boldsymbol{r}, \partial A)>\delta\}
$$

for some $\delta>0$. Consequently, by continuity, we also have that $\tilde{\boldsymbol{\rho}}_{T_{\max }}\left(\mathbb{T}^{d}\right) \subseteq A$. We consider then a solution $\boldsymbol{r}:[0, \varepsilon) \times \mathbb{T}^{d} \rightarrow A, \varepsilon>0$, of the problem (26) starting from $\boldsymbol{r}_{0}=\widetilde{\boldsymbol{\rho}}_{T_{\max }}$ and extend $\rho$ on $\left[0, T_{\max }+\varepsilon\right) \times \mathbb{T}^{d}$ by defining $\rho(t, \cdot):=\boldsymbol{r}\left(t-T_{\max }, \cdot\right)$ for $t \in\left[T_{\max }, T_{\max }+\right.$ $\varepsilon)$. This function is obviously of class $C^{1+\theta, 2+\theta}$ and solves (26), which contradicts the maximality of $T_{\max }$.

\subsection{Proof of Corollary 3.1}

By the global existence in time of solutions to the species-blind parabolic system, it suffices to check that Theorem 3.2 applies. Since the the one-species partition function $\hat{Z}$ is continuous on $\mathcal{D}_{\hat{Z}}$, it follows by the formula $Z(\varphi)=\hat{Z}\left(\varphi_{1}+\varphi_{2}\right)$ that the partition function is continuous. It remains to check that in the case where the associated one-species ZRP has finite critical density, $\boldsymbol{g}$ has regular tails, i.e., that for every $\boldsymbol{v} \in S_{1,+}^{1}$

$$
\mu_{c ; 1}(\boldsymbol{v}):=\log \varphi_{c ; 1}(\boldsymbol{v}):=\liminf _{\substack{|\boldsymbol{k}|_{1} \rightarrow+\infty \\ \boldsymbol{k} /|\boldsymbol{k}|_{1} \rightarrow \boldsymbol{v}}} \frac{1}{|\boldsymbol{k}|_{1}} \log \boldsymbol{g} !(\boldsymbol{k}), \quad \boldsymbol{v} \in S_{1,+}^{1}
$$


exists as a limit and is a continuous function of the direction $\boldsymbol{v} \in S_{1,+}^{1}$. By the formula of $g$ ! we have that

$$
\frac{1}{|\boldsymbol{k}|_{1}} \log \boldsymbol{g} !(\boldsymbol{k})=\frac{1}{|\boldsymbol{k}|_{1}} \log \frac{k_{1} ! k_{2} !}{|\boldsymbol{k}|_{1} !}+\frac{1}{|\boldsymbol{k}|_{1}} \log \hat{g} !\left(|\boldsymbol{k}|_{1}\right) .
$$

The second term in the right hand side of (77) converges as $|\boldsymbol{k}|_{1} \rightarrow+\infty$ to the critical chemical potential $\hat{\mu}_{c}=\log \hat{\varphi}_{c}$ of the one-species jump rate $\hat{g}$. Since by Stirling's approximation $\lim _{k \rightarrow+\infty} \frac{k !}{\sqrt{2 \pi k}(k / e)^{k}}=1$, we can replace the liminf of the first term in the right hand side of (77) by

$$
\liminf _{\substack{|\boldsymbol{k}|_{1} \rightarrow+\infty \\ \boldsymbol{k} /|\boldsymbol{k}|_{1} \rightarrow \boldsymbol{v}}} \frac{1}{|\boldsymbol{k}|_{1}} \log \sqrt{2 \pi} \frac{\sqrt{k_{1}} k_{1}^{k_{1}} \sqrt{k_{2}} k_{2}^{k_{2}}}{\sqrt{|\boldsymbol{k}|_{1}}|\boldsymbol{k}|_{1}^{|\boldsymbol{k}|_{1}}}
$$

This limit inferior exists as a limit and defines a continuous function of $\boldsymbol{v}$. Indeed, for all $\boldsymbol{k} \in \mathbb{N}^{2}$ we have that

$$
\frac{1}{|\boldsymbol{k}|_{1}} \log \frac{\sqrt{k_{1}} k_{1}^{k_{1}} \sqrt{k_{2}} k_{2}^{k_{2}}}{\sqrt{|\boldsymbol{k}|_{1}}|\boldsymbol{k}|_{1}^{|\boldsymbol{k}|_{1}}}=\frac{1}{|\boldsymbol{k}|_{1}} \log \frac{\sqrt{k_{1} k_{2}}}{\sqrt{|\boldsymbol{k}|_{1}}}+\log \left(\frac{k_{1}}{|\boldsymbol{k}|_{1}}\right)^{\frac{k_{1}}{|\boldsymbol{k}|_{1}}}+\log \left(\frac{k_{2}}{|\boldsymbol{k}|_{1}}\right)^{\frac{k_{2}}{|\boldsymbol{k}|_{1}}},
$$

and it is easy to check that $\lim _{|\boldsymbol{k}| \rightarrow+\infty} \frac{1}{|\boldsymbol{k}|_{1}} \log \frac{\sqrt{k_{1} k_{2}}}{\sqrt{|k|_{1}}}=0$, so that

$$
\mu_{c ; 1}(\boldsymbol{v})=\lim _{\substack{|\boldsymbol{k}|_{1} \rightarrow+\infty \\ \boldsymbol{k} /\left.\boldsymbol{k}\right|_{1} \rightarrow \boldsymbol{v} \\ k_{1}, k_{2}>0}}\left[\log \left(\frac{k_{1}}{|\boldsymbol{k}|_{1}}\right)^{\frac{k_{1}}{|\boldsymbol{k}|_{1}}}+\log \left(\frac{k_{2}}{|\boldsymbol{k}|_{1}}\right)^{\frac{k_{2}}{|\boldsymbol{k}|_{1}}}+\log g !\left(|\boldsymbol{k}|_{1}\right)^{\frac{1}{|k|_{1}}}\right]=\langle\boldsymbol{v}, \log \boldsymbol{v}\rangle+\mu_{c}
$$

with the convention $v_{i} \log v_{i}=0$ if $v_{i}=0$ since $x \log x \rightarrow 0$ as $x \rightarrow 0$. Finally, points $\boldsymbol{k} \in \mathbb{N}_{0}^{2}$ with $k_{i}=0$ for some $i=1,2$ contribute to the limit only if $\boldsymbol{v}=\boldsymbol{e}_{i}$ for some $i=1,2$. For such points $\boldsymbol{k} \in \mathbb{N}_{0}^{2}$, we have $k_{1} ! k_{2} !=|\boldsymbol{k}|_{1}$ !, and so the first term in the right hand side of (77) vanishes, which agrees with the fact that $\langle\boldsymbol{v}, \log \boldsymbol{v}\rangle=0$ if $\boldsymbol{v}=\boldsymbol{e}_{i}, i=1,2$. This is to be expected, since in the directions $\boldsymbol{v}=\boldsymbol{e}_{i}$ with $i=1,2$ in the phase space we have only one of the two species of particles, which when on their own perform the underlying one-species ZRP with critical chemical potential $\hat{\mu}_{c}=\log \hat{\varphi}_{c}$. This completes the proof that $g$ has regular tails.

Acknowledgements All authors thank the Leverhulme Trust for its support via Grant RPG-2013-261. JZ gratefully acknowledges funding by the EPSRC through project EP/K027743/1 and a Royal Society Wolfson Research Merit Award. ND gratefully acknowledges funding by the EPSRC through project EP/M028607/1. We also thank the anonymous reviewers for their very careful reading and numerous comments which improved the manuscript.

Open Access This article is distributed under the terms of the Creative Commons Attribution 4.0 International License (http://creativecommons.org/licenses/by/4.0/), which permits unrestricted use, distribution, and reproduction in any medium, provided you give appropriate credit to the original author(s) and the source, provide a link to the Creative Commons license, and indicate if changes were made.

\section{References}

1. Amann, H.: Quasilinear evolution equations and parabolic systems. Trans. Am. Math. Soc. 293(1), 191227 (1986)

2. Arena, O.: A strong maximum principle for quasilinear parabolic differential inequalities. Proc. Am. Math. Soc. 32, 497-502 (1972) 
3. Clément, E.: Hydrodynamic limit for an active exclusion process. arXiv:1608.04937 (2016)

4. Dembo, A., Zeitouni, O.: Large deviations techniques and applications. Stochastic Modelling and Applied Probability, vol. 38. Springer, Berlin (2010). Corrected reprint of the second (1998) edition

5. Duzaar, F., Mingione, G.: Second order parabolic systems, optimal regularity, and singular sets of solutions. Ann. I. H. Poincare 22(6), 705-751 (2005)

6. Evans, L.C.: A strong maximum principle for parabolic systems in a convex set with boundary. Proc. Am. Math. Soc. 138(9), 3179-3185 (2010)

7. Evans, M.R.: Phase transitions in one-dimensional nonequilibrium systems. Braz. J. Phys. 30, 42-57 (2000)

8. Evans, M.R., Hanney, T.: Phase transition in two species zero-range process. J. Phys. A 36(28), L441L447 (2003)

9. Evans, M.R., Hanney, T.: Nonequilibrium statistical mechanics of the zero-range process and related models. J. Phys. A 38(19), R195-R240 (2005)

10. Facchinei, F., Pang, J.S.: Finite-dimensional Variational Inequalities and Complementarity Problems, vol. II. Springer, New York (2003)

11. Godinho, D., Quiñinao, C.: Propagation of chaos for a subcritical Keller-Segel model. Ann. Inst. Henri Poincaré Probab. Stat. 51(3), 965-992 (2015)

12. Grosskinsky, S.: Phase transitions in nonequilibrium stochastic particle systems with local conservation laws. PhD thesis, Technische Universität München (2004)

13. Grosskinsky, S.: Equivalence of ensembles for two-species zero-range invariant measures. Stoch. Process. Appl. 118(8), 1322-1350 (2008)

14. Grosskinsky, S., Marahrens, D., Stevens, A.: A hydrodynamic limit for chemotaxis in a given heterogeneous environment. Vietnam J. Math. 45(1-2), 127-152 (2017)

15. Grosskinsky, S., Schütz, G.M., Spohn, H.: Condensation in the zero range process: stationary and dynamical properties. J. Statist. Phys. 113(3-4), 389-410 (2003)

16. Grosskinsky, S., Spohn, H.: Stationary measures and hydrodynamics of zero range processes with several species of particles. Bull. Braz. Math. Soc. 34(3), 489-507 (2003)

17. Guo, M.Z., Papanicolaou, G.C., Varadhan, S.R.S.: Nonlinear diffusion limit for a system with nearest neighbor interactions. Commun. Math. Phys. 118(1), 31-59 (1988)

18. Hillen, T., Painter, K.J.: A user's guide to PDE models for chemotaxis. J. Math. Biol. 58(1-2), 183-217 (2009)

19. Kipnis, C., Landim, C.: Scaling limits of interacting particle systems. Grundlehren der Mathematischen Wissenschaften [Fundamental Principles of Mathematical Sciences], vol. 320. Springer, Berlin (1999)

20. Lieberman, G.M.: Second Order Parabolic Differential Equations. World Scientific Publishing Co., Inc., River Edge, NJ (1996)

21. Quastel, J.: Diffusion of colour in the simple exclusion process. Commun. Pure Appl. Math 45, 623-679 (1992)

22. Rockafellar, R.T.: Convex Analysis. Princeton Landmarks in Mathematics. Princeton University Press, Princeton, NJ, (1997). Reprint of the 1970 original, Princeton Paperbacks

23. Stamatakis, M.G.: Hydrodynamic limit of mean zero condensing zero range processes with sub-critical initial profiles. J. Stat. Phys. 158(1), 87-104 (2015)

24. Tóth, B., Valkó, B.: Derivation of the Leroux system as the hydrodynamic limit of a two-component latice gas. Commun. Math. Phys. 249, 1-27 (2004)

25. Tóth, B., Valkó, B.: Perturbation of singular equilibria of hyperbolic two-component systems: a universal hydrodynamic limit. Commun. Math. Phys. 256(1), 111-157 (2005)

26. Tsunoda, K.: Hydrodynamic limit for a certain class of two-species zero-range processes. J. Math. Soc. Japan 68(2), 885-898 (2016)

27. Wang, X.: A remark on strong maximum principle for parabolic and elliptic systems. Proc. Am. Math. Soc. 109(2), 343-348 (1990)

28. Yau, H.T.: Relative entropy and hydrodynamics of Ginzburg-Landau models. Lett. Math. Phys. 22(1), 63-80 (1991) 